\title{
The Dynamic Programming Method of Stochastic Differential Game for Functional Forward-Backward Stochastic System
}

\author{
Shaolin Ji, ${ }^{1}$ Chuanfeng Sun, ${ }^{2}$ and Qingmeng $\mathrm{Wei}^{2}$ \\ ${ }^{1}$ Institute for Financial Studies and Institute of Mathematics, Shandong University, Jinan 250100, China \\ ${ }^{2}$ Institute of Mathematics, Shandong University, Jinan 250100, China \\ Correspondence should be addressed to Shaolin Ji; jsl@sdu.edu.cn
}

Received 23 October 2012; Accepted 18 December 2012

Academic Editor: Guangchen Wang

Copyright (c) 2013 Shaolin Ji et al. This is an open access article distributed under the Creative Commons Attribution License, which permits unrestricted use, distribution, and reproduction in any medium, provided the original work is properly cited.

\begin{abstract}
This paper is devoted to a stochastic differential game (SDG) of decoupled functional forward-backward stochastic differential equation (FBSDE). For our SDG, the associated upper and lower value functions of the SDG are defined through the solution of controlled functional backward stochastic differential equations (BSDEs). Applying the Girsanov transformation method introduced by Buckdahn and $\mathrm{Li}$ (2008), the upper and the lower value functions are shown to be deterministic. We also generalize the Hamilton-Jacobi-Bellman-Isaacs (HJBI) equations to the path-dependent ones. By establishing the dynamic programming principal (DPP), we derive that the upper and the lower value functions are the viscosity solutions of the corresponding upper and the lower path-dependent HJBI equations, respectively.
\end{abstract}

\section{Introduction}

The theory of backward stochastic differential equations (BSDEs) has been studied widely since Pardoux and Peng [1] first introduced the nonlinear BSDEs in 1990. BSDEs have got applications in many fields, such as, stochastic control (see Peng [2]), stochastic differential games (SDGs) (see Hamadene and Lepeltier [3], Hamadene et al. [4]), mathematical finance (see El Karoui et al. [5]) and partial differential equation (PDE) theory (see Peng $[6,7]$ ), and so forth.

In the aspect of finance, the BSDE theory presents a simple formulation of stochastic differential utilities introduced by Duffie and Epstein [8]. When the generator $g$ of a BSDE does not depend on $z$, the solution $Y$ is just the comparison theorem for BSDEs, we know

recursive utility presented in [8]. From the view of BSDE, by studying some important properties (such as, comparison theorem) of BSDEs, El Karoui et al. [5] gave the more general class of recursive utilities and their properties. And later the recursive optimal control problems whose cost functionals are described by the solution of BSDE are studied widely. Peng
[7] obtained the Bellman's dynamic programming principle (DPP) for this kind of problem and proved the value function to be a viscosity solution of one kind of quasi-linear second order PDE, that is, Hamilton-Jacobi-Bellman (HJB) equation. Later, for the recursive optimal control problem introduced by a BSDE under Markovian framework, by introducing the notion of backward semigroup of BSDE, in Peng [9], the Bellman's DPP is derived and the value function is proved to be a viscosity solution of a generalized HJB equation.

By now, the DPP with related HJB equation has become a powerful approach to solving optimal control and game problems (see [10-13]). In [10], Buckdahn and Li studied a recursive SDG problem and interpreted the relationship between the controlled system and the Hamilton-JacobiBellman-Isaacs (HJBI) equation. A point is worthy to mention: in order to derive the DPP, they introduced a Girsanov transformation method to prove the value functions are deterministic which is different from the method developed in Peng [9].

There really exist some systems which are modeled only by stochastic systems whose evolutions depend on the past history of the states. Based on this phenomenon, Ji and Yang 
[14] investigated a controlled system governed by a functional forward-backward stochastic differential equation (FBSDE) and proved the value function to be the viscosity solution of the related path-dependent HJB equation.

In this paper, inspired by $[10,14]$, we will investigate the SDG problems of the functional FBSDEs. Precisely, the dynamics of our SDGs is described by the following functional SDE:

$$
\begin{aligned}
d X^{\gamma_{t} ; u, v}(s)= & b\left(X_{s}^{\gamma_{t} ; u, v}, u(s), v(s)\right) d s \\
& +\sigma\left(X_{s}^{\gamma_{t}} ; u, v\right. \\
X_{t}^{\gamma_{t}, u, v} & u(s), v(s)) d B(s), \quad s \in[t, T],
\end{aligned}
$$

And the cost functional $J\left(\gamma_{t} ; u, v\right)$ is defined as $Y^{\gamma_{t} ; u, v}(t)$ which is the solution of the following functional BSDE:

$$
\begin{gathered}
d Y^{\gamma_{t} ; u, v}(s)=-f\left(X_{s}^{\gamma_{t} ; u, v}, Y^{\gamma_{t} ; u, v}(s), Z^{\gamma_{t} ; u, v}(s),\right. \\
u(s), v(s)) d s+Z^{\gamma_{t} ; u, v}(s) d B(s), \\
Y^{\gamma_{t}, u, v}(T)=\Phi\left(X_{T}^{\gamma_{t} ; u, v}\right), \quad s \in[t, T],
\end{gathered}
$$

where $\gamma_{t}$ is a path on $[0, t]$. The driver $f$ and $\Phi$ can be interpreted as the running cost and the terminal cost, respectively. Also, they depend on the past history of the dynamics. Equations (1) and (2) compose a decoupled functional FBSDE. The concrete conditions on $b, \sigma, f, \Phi$ are shown in the later section.

In the context, we adopt the strategy against control form. The cost functional $J\left(\gamma_{t} ; u, v\right)$ is explained as a payoff for player I and as a cost for player II. The aim of this paper is to show the following lower and upper value functions:

$$
\begin{gathered}
W\left(\gamma_{t}\right):=\underset{\beta \in \mathscr{B}_{t, T}}{\operatorname{essinf}} \operatorname{esssup} J\left(\gamma_{t} ; u, \beta(u)\right), \\
U\left(\gamma_{t}\right):=\underset{\alpha \in \mathscr{A}_{t, T}}{\operatorname{esssup}} \operatorname{essinf}_{v \in \mathscr{V}_{t, T}} J\left(\gamma_{t} ; \alpha(v), v\right)
\end{gathered}
$$

are the viscosity solutions of the following path-dependent $\mathrm{HJBI}$ equations, respectively,

$$
\begin{gathered}
D_{t} W\left(\gamma_{t}\right)+\sup _{u \in U} \inf _{v \in V} H\left(\gamma_{t}, W, D_{x} W, D_{x x} W, u, v\right)=0, \\
W\left(\gamma_{T}\right)=\Phi\left(\gamma_{T}\right), \quad \gamma_{T} \in \Lambda, \\
D_{t} U\left(\gamma_{t}\right)+\inf _{v \in V} \sup _{u \in U} H\left(\gamma_{t}, U, D_{x} U, D_{x x} U, u, v\right)=0, \\
U\left(\gamma_{t}\right)=\Phi\left(\gamma_{T}\right), \quad \gamma_{T} \in \Lambda,
\end{gathered}
$$

where

$$
\begin{aligned}
H\left(\gamma_{t}, y, p, X, u, v\right)= & \frac{1}{2} \operatorname{tr}\left(\sigma \sigma^{T}\left(\gamma_{t}, u, v\right) X\right) \\
& +p \cdot b\left(\gamma_{t}, u, v\right) \\
& +f\left(\gamma_{t}, y, p \cdot \sigma\left(\gamma_{t}, u, v\right), u, v\right),
\end{aligned}
$$

where $\left(\gamma_{t}, y, p, X\right) \in \Lambda \times \mathbb{R} \times \mathbb{R}^{d} \times \mathbb{S}^{d}\left(\mathbb{S}^{d}\right.$ denotes the set of $d \times d$ symmetric matrices).

To solve the above SDG, we need the functional Itô's calculus and path-dependent PDEs which are recently introduced by Dupire [15] (for a recent account of this theory, the reader may consult [16-18]. And under the framework of functional Itô's calculus, for the non-Markovian BSDEs, Peng and Wang [19] derived a nonlinear Feynman-Kac formula for classical solutions of path-dependent PDEs. For the further development, the readers may refer to $[20,21])$.

In this paper, we apply the Girsanov transformation method in Buckdahn and $\mathrm{Li}$ [10] to prove the determinacy of the value functions, which is different from the method introduced by Peng [7, 9]. Making use of this method and the functional Itô's calculus (introduced by Dupire [15] and developed by Cont and Fournié [16-18]), we complete the study of the zero-sum two-player SDGs in the nonMarkovian case and present the lower and upper value functions of our SDG are the viscosity solutions of the corresponding path-dependent HJBI equations, respectively.

Different from the HJBI equations developed for stochastic delay systems, we establish the DPP and derive the HJBI equation in the new framework of functional Itô's calculus.

This paper is organized as follows. Section 2 recalls the functional Itô's calculus and the well-known results of BSDEs we will use later. In Section 3, we formulate our SDGs and get the corresponding DPP. Based on the obtained DPP, in Section 4 we derive the main result of the paper: the lower and upper value functions are the viscosity solutions of the associated path-dependent HJBI equations, respectively. And we add the proof for the DPP in the appendix.

\section{Preliminaries}

2.1. Functional Itô's Calculus. We present some preliminaries for functional Itô's calculus introduced firstly by Dupire [15]. Here we follow the notations in [15].

Let $T>0$ be fixed. For each $t \in[0, T]$, we denote $\Lambda_{t}$ the set of càdlàg functions from $[0, t]$ to $\mathbb{R}^{d}$.

For $\gamma \in \Lambda_{T}$, denote $\gamma(s)$ by the value of $\gamma$ at time $s \in[0, T]$. Thus $\gamma=(\gamma(s))_{0 \leq s \leq T}$ is a càdlàg process on $[0, T]$ and its value at time $s$ is $\gamma(s) \cdot \gamma_{t}=(\gamma(s))_{0 \leq s \leq t} \in \Lambda_{t}$ is the path of $\gamma$ up to time $t$. We denote $\Lambda=\bigcup_{t \in[0, T]} \Lambda_{t}$. For each $\gamma_{t} \in \Lambda$ and $x \in \mathbb{R}^{d}, \gamma_{t}(s)$ is denoted by the value of $\gamma_{t}$ at $s \in[0, t]$ and $\gamma_{t}^{x}:=\left(\gamma_{t}(s)_{0 \leq s<t}, \gamma_{t}(t)+x\right)$ which is also an element in $\Lambda_{t}$.

We now introduce a distance on $\Lambda$. Let $\langle\cdot, \cdot\rangle$ and $|\cdot|$ denote the inner product and norm in $\mathbb{R}^{d}$. For each $0 \leq t, \bar{t} \leq T$ and $\gamma_{t}, \gamma_{\bar{t}} \in \Lambda$, we set

$$
\begin{gathered}
\left\|\gamma_{t}\right\|:=\sup _{s \in[0, t]}\left|\gamma_{t}(s)\right|, \\
\left\|\gamma_{t}-\bar{\gamma}_{\bar{t}}\right\|:=\sup _{s \in[0, t \vee \bar{t}]}\left|\gamma_{t}(s \wedge t)-\bar{\gamma}_{\bar{t}}(s \wedge \bar{t})\right|, \\
d_{\infty}\left(\gamma_{t}, \bar{\gamma}_{\bar{t}}\right):=\sup _{s \in[0, t \vee \bar{t}]}\left|\gamma_{t}(s \wedge t)-\bar{\gamma}_{\bar{t}}(s \wedge \bar{t})\right|+|t-\bar{t}| .
\end{gathered}
$$

It is obvious that $\Lambda_{t}$ is a Banach space with respect to $\|\cdot\|$. Since $\Lambda$ is not a linear space, $d_{\infty}$ is not a norm. 
Definition 1. A functional $u: \Lambda \mapsto \mathbb{R}$ is $\Lambda$-continuous at $\gamma_{t} \in \Lambda$, if for any $\varepsilon>0$ there exists $\delta>0$ such that for each $\bar{\gamma}_{\bar{t}} \in \Lambda$ with $d_{\infty}\left(\gamma_{t}, \bar{\gamma}_{\bar{t}}\right)<\delta$, one has $\left|u\left(\gamma_{t}\right)-u\left(\bar{\gamma}_{\bar{t}}\right)\right|<\varepsilon$.

$u$ is said to be $\Lambda$-continuous if it is $\Lambda$-continuous at each $\gamma_{t} \in \Lambda$.

Definition 2. Let $v: \Lambda \mapsto \mathbb{R}$ and $\gamma_{t} \in \Lambda$ be given. If there exists $p \in \mathbb{R}^{d}$, such that

$$
v\left(\gamma_{t}^{x}\right)=v\left(\gamma_{t}\right)+\langle p, x\rangle+o(|x|), \quad \text { as } x \longrightarrow 0, x \in \mathbb{R}^{d} .
$$

Then we say that $v$ is (vertically) differentiable at $t$ and denote the gradient of $D_{x} v\left(\gamma_{t}\right)=p \cdot v$ is said to be vertically differentiable in $\Lambda$, if $D_{x} v\left(\gamma_{t}\right)$ exists for each $\gamma_{t} \in \Lambda$. The Hessian $D_{x x} v\left(\gamma_{t}\right)$ can be defined similarly. It is an $S(d)$-valued function defined on $\Lambda$, where $S(d)$ is the space of all $d \times d$ symmetric matrices.

For each $\gamma_{t} \in \Lambda$, we denote $\gamma_{t, s}(r):=\gamma_{t}(r) 1_{[0, t)}(r)+$ $\gamma_{t}(t) 1_{[t, s]}(r), r \in[0, s]$. It is clear that $\gamma_{t, s} \in \Lambda_{s}$.

Definition 3. For a given $\gamma_{t} \in \Lambda$, if one has

$$
v\left(\gamma_{t, s}\right)=v\left(\gamma_{t}\right)+a(s-t)+o(|s-t|), \quad \text { as } s \longrightarrow t, s \geq t,
$$

then we say that $v\left(\gamma_{t}\right)$ is (horizontally) differentiable in $t$ at $\gamma_{t}$ and denote $D_{t} v\left(\gamma_{t}\right)=a . v$ is said to be horizontally differentiable in $\Lambda$ if $D_{t} v\left(\gamma_{t}\right)$ exists for each $\gamma_{t} \in \Lambda$.

Definition 4. Define $\mathbb{C}^{j, k}(\Lambda)$ as the set of function $v:=$ $\left(v\left(\gamma_{t}\right)\right)_{\gamma_{t} \in \Lambda}$ defined on $\Lambda$ which are $j$ times horizontally and $k$ times vertically differentiable in $\Lambda$ such that all these derivatives are $\Lambda$-continuous.

The following is about the functional Itô's formula which was firstly obtained by Dupire [15] and then developed by Cont and Fournié [18] for a more general formulation.

Theorem 5 (functional Itô's formula). Let $\left(\Omega, \mathscr{F},\left(\mathscr{F}_{t}\right)_{t \in[0, T}\right]$, $P)$ be a probability space, if $X$ is a continuous semimartingale and $v$ is in $\mathbb{C}^{1,2}(\Lambda)$, then for any $t \in[0, T)$,

$$
\begin{aligned}
v\left(X_{t}\right)-v\left(X_{0}\right)= & \int_{0}^{t} D_{s} v\left(X_{s}\right) d s+\int_{0}^{t} D_{x} v\left(X_{s}\right) d X(s) \\
& +\frac{1}{2} \int_{0}^{t} D_{x x} v\left(X_{s}\right) d\langle X\rangle(s), \quad P \text {-a.s. }
\end{aligned}
$$

2.2. BSDEs. In this section, we recollect some important results which will be used in our SDG problems.

Let $(\Omega, \mathscr{F}, P)$ be the Wiener space, where $\Omega$ is the set of continuous functions from $[0, T]$ to $\mathbb{R}^{d}$ starting from $0(\Omega=$ $\left.C_{0}\left([0, T] ; \mathbb{R}^{d}\right)\right), T$ is an arbitrarily fixed real time horizon, $\mathscr{F}$ is the completed Borel $\sigma$-algebra over $\Omega$, and $P$ is the Wiener measure. Let $B$ be the canonical process: $B(\omega, s)=\omega_{s}, s \in$ $[0, T], \omega \in \Omega$. We denote by $\mathbb{F}=\left\{\mathscr{F}_{s}, 0 \leq s \leq T\right\}$ the natural filtration generated by $\{B(t)\}_{t \geq 0}$ and augmented by all $P$-null sets, that is, $\mathscr{F}_{s}=\sigma\{B(r), r \leq s\} \vee \mathscr{N}_{P}, s \in[0, T] ; \mathscr{F}_{t}^{s}=$ $\sigma(B(r)-B(t), t \leq r \leq s) \vee \mathcal{N}_{P}$, where $\mathcal{N}_{P}$ is the set of all $P$-null subsets. First we present two spaces of processes as follows:

$$
\mathcal{S}^{2}\left(0, T ; \mathbb{R}^{n}\right):=\left\{(\psi(t))_{0 \leq t \leq T} \mathbb{R}^{n}\right. \text {-valued }
$$

$\mathbb{F}$-adapted continuous process :

$$
\begin{array}{r}
\left.E\left[\sup _{0 \leq t \leq T}|\psi(t)|^{2}\right]<+\infty\right\} ; \\
\mathscr{H}^{2}\left(0, T ; \mathbb{R}^{n}\right):=\left\{(\psi(t))_{0 \leq t \leq T} \mathbb{R}^{n}\right. \text {-valued }
\end{array}
$$

F-progressively measurable process :

$$
\left.\|\psi\|^{2}=E\left[\int_{0}^{T}|\psi(t)|^{2} d t\right]<+\infty\right\} .
$$

Consider $g: \Omega \times[0, T] \times \mathbb{R} \times \mathbb{R}^{d} \rightarrow \mathbb{R}$, for every $(y, z)$ in $\mathbb{R} \times \mathbb{R}^{d},(g(t, y, z))_{t \in[0, T]}$ is progressively measurable and satisfies the following conditions:

(A1) there exists a constant $C \geq 0$ such that, $P$-a.s., for all $t \in[0, T], y_{1}, y_{2} \in \mathbb{R}, z_{1}, z_{2} \in \mathbb{R}^{d}$,

$\left|g\left(t, y_{1}, z_{1}\right)-g\left(t, y_{2}, z_{2}\right)\right| \leq C\left(\left|y_{1}-y_{2}\right|+\left|z_{1}-z_{2}\right|\right)$,

(A2) $g(\cdot, 0,0) \in \mathscr{H}^{2}(0, T ; \mathbb{R})$.

In the following, we suppose the driver $g$ of a BSDE satisfies (A1) and (A2).

Lemma 6. Under the assumptions (A1) and (A2), for any random variable $\xi \in L^{2}\left(\Omega, \mathscr{F}_{T}, P ; \mathbb{R}\right)$, the BSDE

$$
\begin{array}{r}
y(t)=\xi+\int_{t}^{T} g(s, y(s), z(s)) d s-\int_{t}^{T} z(s) d B(s), \\
0 \leq t \leq T
\end{array}
$$

has a unique adapted solution

$$
(y(t), z(t))_{t \in[0, T]} \in \mathcal{S}^{2}(0, T ; \mathbb{R}) \times \mathscr{H}^{2}\left(0, T ; \mathbb{R}^{d}\right) .
$$

The readers may refer to Pardoux and Peng [1] for the above well-known existence and uniqueness results on BSDEs.

Lemma 7 (comparison theorem). Given two coefficients $g_{1}$, $g_{2}$ satisfying $(A 1),(A 2)$, and two terminal values $\xi_{1}, \xi_{2} \epsilon$ $L^{2}\left(\Omega, \mathscr{F}_{T}, P ; \mathbb{R}\right)$, by $\left(y^{1}, z^{1}\right)$ and $\left(y^{2}, z^{2}\right)$ one denotes the solution of a BSDE with the data $\left(\xi_{1}, g_{1}\right)$ and $\left(\xi_{2}, g_{2}\right)$, respectively. Then one has the following.

(i) If $\xi_{1} \geq \xi_{2}$ and $g_{1} \geq g_{2}$,P-a.s., then $y^{1}(t) \geq y^{2}(t)$, $P$-a.s., for all $t \in[0, T]$. 
(ii) (Strict monotonicity) If, in addition to (i), one also assumes that $P\left(\xi_{1}>\xi_{2}\right)>0$, then $P\left(y^{1}(t)>y^{2}(t)\right)>$ $0,0 \leq t \leq T$, and, in particular, $y^{1}(0)>y^{2}(0)$.

With the notations in Lemma 7, we assume that, for some $g: \Omega \times[0, T] \times \mathbb{R} \times \mathbb{R}^{d} \rightarrow \mathbb{R}$ satisfying (A1) and (A2), the drivers $g_{i}$ have the following form:

$$
\begin{array}{r}
g_{i}\left(s, y^{i}(s), z^{i}(s)\right)=g\left(s, y^{i}(s), z^{i}(s)\right)+\varphi_{i}(s), \\
d s d P \text {-a.e., } \quad i=1,2,
\end{array}
$$

where $\varphi_{i} \in \mathscr{H}^{2}(0, T ; \mathbb{R})$. Then, the following results hold true for all terminal values $\xi_{1}, \xi_{2} \in L^{2}\left(\Omega, \mathscr{F}_{T}, P ; \mathbb{R}\right)$.

Lemma 8. The difference of the solutions $\left(y^{1}, z^{1}\right)$ and $\left(y^{2}, z^{2}\right)$ of BSDE (12) with the data $\left(\xi_{1}, g_{1}\right)$ and $\left(\xi_{2}, g_{2}\right)$, respectively, satisfies the following estimate:

$$
\begin{gathered}
\left|y^{1}(t)-y^{2}(t)\right|^{2}+\frac{1}{2} E\left[\int _ { t } ^ { T } e ^ { \beta ( s - t ) } \left(\left|y^{1}(s)-y^{2}(s)\right|^{2}\right.\right. \\
\left.\left.+\left|z^{1}(s)-z^{2}(s)\right|^{2}\right) d s \mid \mathscr{F}_{t}\right] \\
\leq E\left[e^{\beta(T-t)}\left|\xi_{1}-\xi_{2}\right|^{2} \mid \mathscr{F}_{t}\right] \\
+E\left[\int_{t}^{T} e^{\beta(s-t)}\left|\varphi_{1}(s)-\varphi_{2}(s)\right|^{2} d s \mid \mathscr{F}_{t}\right] \\
P \text {-a.s., } \forall 0 \leq t \leq T, \text { where } \beta=16\left(1+C^{2}\right) .
\end{gathered}
$$

Proof. The reader may refer to Proposition 2.1 in El Karoui et al. [5] or Theorem 2.3 in Peng [9] for the details.

\section{A DPP for Stochastic Differential Games of Functional FBSDEs}

In this section, we consider the SDGs of functional FBSDEs.

First we introduce the background of SDGs. Suppose that the control state spaces $U, V$ are compact metric spaces. $\mathscr{U}$ (resp., $\mathscr{V}$ ) is the control set of all $U$ (resp., $V$ )-valued $\mathbb{F}$-progressively measurable processes for the first (resp., second) player. If $u \in \mathscr{U}$ (resp., $v \in \mathscr{V}$ ), we call $u$ (resp., $v$ ) an admissible control.

Let us give the following mappings:

$$
\begin{gathered}
b: \Lambda \times U \times V \longrightarrow \mathbb{R}^{n}, \\
\sigma: \Lambda \times U \times V \longrightarrow \mathbb{R}^{n \times d}, \\
f: \Lambda \times \mathbb{R} \times \mathbb{R}^{d} \times U \times V \longrightarrow \mathbb{R} .
\end{gathered}
$$

For given admissible controls $u(\cdot) \in \mathscr{U}, v(\cdot) \in \mathscr{V}$, and $t \epsilon$ $[0, T], \gamma_{t} \in \Lambda$, we consider the following functional forwardbackward stochastic system:

$$
\begin{aligned}
& d X^{\gamma_{t} ; u, v}(s)=b\left(X_{s}^{\gamma_{t} ; u, v}, u(s), v(s)\right) d s \\
& +\sigma\left(X_{s}^{\gamma_{t} ; u, v}, u(s), v(s)\right) d B(s), \quad s \in[t, T], \\
& d Y^{\gamma_{t} ; u, v}(s)=-f\left(X_{s}^{\gamma_{t} ; u, v}, Y^{\gamma_{t} ; u, v}(s), Z^{\gamma_{t} ; u, v}(s), u(s), v(s)\right) d s \\
& +Z^{\gamma_{t} ; u, v}(s) d B(s) \\
& X_{t}^{\gamma_{t}, u, v}=\gamma_{t} \\
& Y^{\gamma_{t}, u, v}(T)=\Phi\left(X_{T}^{\gamma_{t} ; u, v}\right)
\end{aligned}
$$

(H) (i) For all $t \in[0, T], u \in U, v \in V, x_{t} \in \Lambda, y \in$ $\mathbb{R}, z \in \mathbb{R}^{d}, b\left(x_{t}, u, v\right), \sigma\left(x_{t}, u, v\right)$, and $f\left(x_{t}, y, z, u, v\right)$ are $\mathscr{F}_{t}$-measurable.

(ii) There exists a constant $C>0$, such that, for all $t \in$ $[0, T], u \in U, v \in V$, for any $x_{t}^{1}, x_{t}^{2} \in \Lambda$,

$$
\begin{aligned}
& \left|b\left(x_{t}^{1}, u, v\right)-b\left(x_{t}^{2}, u, v\right)\right|+\left|\sigma\left(x_{t}^{1}, u, v\right)-\sigma\left(x_{t}^{2}, u, v\right)\right| \\
& \leq C\left\|x_{t}^{1}-x_{t}^{2}\right\|,
\end{aligned}
$$

$\left|b\left(x_{t}, u, v\right)\right|+\left|\sigma\left(x_{t}, u, v\right)\right| \leq C\left(1+\left\|x_{t}\right\|\right)$, for any $x_{t} \in \Lambda$.

(iii) There exists a constant $C>0$, such that for all $t \in$ $[0, T], u \in U, v \in V$, for any $x_{t}^{1}, x_{t}^{2} \in \Lambda, y^{1}, y^{2} \in$ $\mathbb{R}, z^{1}, z^{2} \in \mathbb{R}^{d}$

$$
\begin{gathered}
\left|f\left(x_{t}^{1}, y^{1}, z^{1}, u, v\right)-f\left(x_{t}^{2}, y^{2}, z^{2}, u, v\right)\right| \\
\leq C\left(\left\|x_{t}^{1}-x_{t}^{2}\right\|+\left|y^{1}-y^{2}\right|+\left|z^{1}-z^{2}\right|\right), \\
\left|\Phi\left(x_{T}^{1}\right)-\Phi\left(x_{T}^{2}\right)\right| \leq C\left\|x_{T}^{1}-x_{T}^{2}\right\|, \\
\left|f\left(x_{t}, 0,0, u, v\right)\right| \leq C\left(1+\left\|x_{t}\right\|\right), \\
\left|\Phi\left(x_{T}\right)\right| \leq C\left(1+\left\|x_{T}\right\|\right), \quad \text { for any } x_{t} \in \Lambda .
\end{gathered}
$$

Theorem 9. Under the assumption $(\mathrm{H})$, there exists a unique solution $(X, Y, Z) \in \mathcal{S}^{2}\left(0, T ; \mathbb{R}^{n}\right) \times \mathcal{S}^{2}(0, T ; \mathbb{R}) \times \mathscr{H}^{2}\left(0, T ; \mathbb{R}^{d}\right)$ solving (17).

We recall the subspaces of admissible controls and the definitions of admissible strategies as follows, which are similar to [10].

Definition 10. An admissible control process $u=\left(u_{r}\right)_{r \in[t, s]}$ (resp., $v=\left(v_{r}\right)_{r \in[t, s]}$ ) for Player I (resp., II) on $[t, s]$ is an $\mathscr{F}_{r}{ }^{-}$ progressively measurable, $U$ (resp., $V$ )-valued process. The set of all admissible controls for Player I (resp., II) on $[t, s]$ is denoted by $\mathscr{U}_{t, s}$ (resp., $\mathscr{V}_{t, s}$ ). If $P\{u \equiv \bar{u}$,a.e., in $[t, s]\}=1$, one will identify both processes $u$ and $\bar{u}$ in $\mathscr{U}_{t, s}$. Similarly one interprets $v \equiv \bar{v}$ on $[t, s]$ in $\mathscr{V}_{t, s}$. 
Definition 11. A nonanticipative strategy for Player I on $[t, s](t<s \leq T)$ is a mapping $\alpha: \mathscr{V}_{t, s} \rightarrow \mathscr{U}_{t, s}$ such that, for any $\mathbb{F}$-stopping time $S: \Omega \rightarrow[t, s]$ and any $v_{1}, v_{2} \in \mathscr{V}_{t, s}$, with $v_{1} \equiv v_{2}$ on $[[t, S]]$, it holds that $\alpha\left(v_{1}\right) \equiv$ $\alpha\left(v_{2}\right)$ on $[[t, S]]$. Nonanticipative strategies for Player II on $[t, s], \beta: \mathscr{U}_{t, s} \rightarrow \mathscr{V}_{t, s}$, are defined similarly. The set of all nonanticipative strategies $\alpha: \mathscr{V}_{t, s} \rightarrow \mathscr{U}_{t, s}$ for Player I on $[t, s]$ is denoted by $\mathscr{A}_{t, s}$. The set of all nonanticipative strategies $\beta: \mathscr{U}_{t, s} \rightarrow \mathscr{V}_{t, s}$ for Player II on $[t, s]$ is denoted by $\mathscr{B}_{t, S^{*}}($ Recall that $[[t, S]]=\{(r, \omega) \in[0, T] \times \Omega, t \leq r \leq S(\omega)$.)

For given processes $u(\cdot) \in \mathscr{U}_{t, T}, v(\cdot) \in \mathscr{V}_{t, T}$, initial data $t \in[0, T], \gamma_{t} \in \Lambda$, the cost functional is defined as follows:

$$
J\left(\gamma_{t} ; u, v\right):=Y^{\gamma_{t} ; u, v}(t), \quad \gamma_{t} \in \Lambda
$$

where the process $Y^{\gamma_{t} ; u, v}$ is defined by functional FBSDE (17).

For $\gamma_{t} \in \Lambda$, the lower and the upper value functions of our SDGs are defined as

$$
\begin{aligned}
& W\left(\gamma_{t}\right):=\underset{\beta \in \mathscr{B}_{t, T}}{\operatorname{essinf}} \underset{u \in \mathscr{U}_{t, T}}{\operatorname{esssup}} J\left(\gamma_{t} ; u, \beta(u)\right), \\
& U\left(\gamma_{t}\right):=\operatorname{esssup}_{\alpha \in \mathscr{A}_{t, T}}^{\operatorname{essinf}} \underset{v \in \mathscr{V}_{t, T}}{\operatorname{essin}}\left(\gamma_{t} ; \alpha(v), v\right) .
\end{aligned}
$$

As we know, the essential infimum and essential supremum on a family of random variables are still random variables. But by applying the method introduced by Buckdahn and $\mathrm{Li}[10]$, we get $W\left(\gamma_{t}\right)$ and $U\left(\gamma_{t}\right)$ are deterministic.

Proposition 12. For any $t \in[0, T], \gamma_{t} \in \Lambda, W\left(\gamma_{t}\right)$ is a deterministic function in the sense that $W\left(\gamma_{t}\right)=E\left[W\left(\gamma_{t}\right)\right], P$-a.s.

Proof. Let $H$ denote the Cameron-Martin space of all absolutely continuous elements $h \in \Omega$ whose derivative $h$ belongs to $L^{2}\left([0, T] ; \mathbb{R}^{d}\right)$.

For any $h \in H$, we define the mapping $\tau_{h} \omega:=\omega+h, \omega \in$ $\Omega$. It is easy to check that $\tau_{h}: \Omega \rightarrow \Omega$ is a bijection, and its law is given by $P \circ\left[\tau_{h}\right]^{-1}=\exp \left\{\int_{0}^{T} \dot{h}(s) d B(s)-\right.$ $\left.(1 / 2) \int_{0}^{T}|\dot{h}(s)|^{2} d s\right\} P$. For any fixed $t \in[0, T]$, set $H_{t}=\{h \in$ $H \mid h(\cdot)=h(\cdot \wedge t)\}$. The proof can be separated into the following four steps.

(1) For all $u \in \mathscr{U}_{t, T}, v \in \mathscr{V}_{t, T}, h \in H_{t}, \quad J\left(\gamma_{t} ; u, v\right)\left(\tau_{h}\right)=$ $J\left(\gamma_{t} ; u\left(\tau_{h}\right), v\left(\tau_{h}\right)\right), P$-a.s.

First, we make the transformation for the functional SDE as follows:

$$
\begin{gathered}
X^{\gamma_{t} ; u, v}(s) \circ\left(\tau_{h}\right)=\left(\gamma_{t}(t)+\int_{t}^{s} b\left(X_{r}^{\gamma_{t} ; u, v}, u(r), v(r)\right) d r\right. \\
\left.\quad+\int_{t}^{s} \sigma\left(X_{r}^{\gamma_{t} ; u, v}, u(r), v(r)\right) d B(r)\right) \circ\left(\tau_{h}\right) \\
=\gamma_{t}(t)+\int_{t}^{s} b\left(X_{r}^{\gamma_{t} ; u, v}\left(\tau_{h}\right), u\left(\tau_{h}\right)(r)\right. \\
\left.v\left(\tau_{h}\right)(r)\right) d r
\end{gathered}
$$

$$
\begin{gathered}
+\int_{t}^{s} \sigma\left(X_{r}^{\gamma_{t}} ; u, v\left(\tau_{h}\right), u\left(\tau_{h}\right)(r),\right. \\
\left.v\left(\tau_{h}\right)(r)\right) d B(r), \\
X^{\gamma_{t} ; u\left(\tau_{h}\right), v\left(\tau_{h}\right)}(s)=\gamma_{t}(t)+\int_{t}^{s} b\left(X_{r}^{\gamma_{t} ; u\left(\tau_{h}\right), v\left(\tau_{h}\right)}, u\left(\tau_{h}\right)(r),\right. \\
\left.v\left(\tau_{h}\right)(r)\right) d r \\
+\int_{t}^{s} \sigma\left(X_{r}^{\gamma_{t} ; u\left(\tau_{h}\right), v\left(\tau_{h}\right)}, u\left(\tau_{h}\right)(r),\right. \\
\left.v\left(\tau_{h}\right)(r)\right) d B(r),
\end{gathered}
$$

then, from the uniqueness of the solution of the functional $\mathrm{SDE}$, we get

$$
\begin{array}{r}
X^{\gamma_{t} ; u, v}(s)\left(\tau_{h}\right)=X^{\gamma_{t} ; \mathcal{u}\left(\tau_{h}\right), v\left(\tau_{h}\right)}(s), \\
\text { for any } s \in[t, T], P \text {-a.s. }
\end{array}
$$

Similarly, using the transformation to the BSDE in (17) and comparing the obtained equation with the BSDE obtained from (17) by replacing the transformed control process $u\left(\tau_{h}\right), v\left(\tau_{h}\right)$ for $u, v$, due to the uniqueness of the solution of functional BSDE, we obtain

$$
\begin{array}{r}
Y^{\gamma_{t} ; u, v}(s)\left(\tau_{h}\right)=Y^{\gamma_{t} ; u\left(\tau_{h}\right), v\left(\tau_{h}\right)}(s), \\
\text { for any } s \in[t, T], P \text {-a.s., } \\
Z^{\gamma_{t} ; u, v}(s)\left(\tau_{h}\right)=Z^{\gamma_{t} ; u\left(\tau_{h}\right), v\left(\tau_{h}\right)}(s), \\
d s d P \text {-a.e. on }[0, T] \times \Omega .
\end{array}
$$

Hence

$$
J\left(\gamma_{t} ; u, v\right)\left(\tau_{h}\right)=J\left(\gamma_{t} ; u\left(\tau_{h}\right), v\left(\tau_{h}\right)\right), \quad P \text {-a.s. }
$$

(2) For $\beta \in \mathscr{B}_{t, T}, h \in H_{t}$, let $\beta^{h}(u):=\beta\left(u\left(\tau_{-h}\right)\right)\left(\tau_{h}\right), u \in$ $\mathcal{U}_{t, T}$. Then, $\beta^{h} \in \mathscr{B}_{t, T}$.

Obviously, $\beta^{h}: \mathscr{U}_{t, T} \rightarrow \mathscr{V}_{t, T}$. And it is nonanticipating. In fact, given an $\mathbb{F}$-stopping time $S: \Omega \rightarrow[t, T]$ and $u_{1}, u_{2} \in$ $\mathcal{u}_{t, T}$, with $u_{1} \equiv u_{2}$ on $[[t, S]]$. Accordingly, $u_{1}\left(\tau_{-h}\right) \equiv u_{2}\left(\tau_{-h}\right)$ on $\left[\left[t, S\left(\tau_{-h}\right)\right]\right]$. Thus,

$$
\begin{aligned}
\beta^{h}\left(u_{1}\right) & =\beta\left(u_{1}\left(\tau_{-h}\right)\right)\left(\tau_{h}\right)=\beta\left(u_{2}\left(\tau_{-h}\right)\right)\left(\tau_{h}\right) \\
& =\beta^{h}\left(u_{2}\right) \text { on }[[t, S]] .
\end{aligned}
$$

(3) For any $h \in H_{t}$, and $\beta \in \mathscr{B}_{t, T}$, we have

$$
\begin{aligned}
& \left\{\operatorname{esssup}_{u \in \mathscr{U}_{t, T}} J\left(\gamma_{t} ; u, \beta(u)\right)\right\}\left(\tau_{h}\right) \\
& =\underset{u \in \mathcal{U}_{t, T}}{\operatorname{esssup}}\left\{J\left(\gamma_{t} ; u, \beta(u)\right)\left(\tau_{h}\right)\right\}, \quad P \text {-a.s. }
\end{aligned}
$$

In fact, for convenience, setting $I\left(\gamma_{t} ; \beta\right):=\operatorname{esssup}_{u \in \mathcal{U}_{t, T}} J\left(\gamma_{t} ; u\right.$, $\beta(u)), \beta \in \mathscr{B}_{t, T}$, we know $I\left(\gamma_{t} ; \beta\right) \geq J\left(\gamma_{t} ; u, \beta(u)\right)$. Then 
$I\left(\gamma_{t} ; \beta\right)\left(\tau_{h}\right) \geq J\left(\gamma_{t} ; u, \beta(u)\right)\left(\tau_{h}\right), P$-a.s., for all $u \in \mathscr{U}_{t, T}$. Therefore,

$$
\begin{aligned}
& \left\{\operatorname{lissup}_{u \in \mathscr{U}_{t, T}}^{\operatorname{essup}} J\left(\gamma_{t} ; u, \beta(u)\right)\right\}\left(\tau_{h}\right) \\
& \quad \geq \operatorname{esssup}_{u \in \mathscr{U}_{t, T}}\left\{J\left(\gamma_{t} ; u, \beta(u)\right)\left(\tau_{h}\right)\right\}, \quad P \text {-a.s. }
\end{aligned}
$$

From the definition of essential supremum, for any random variable $\xi$ which satisfies $\xi \geq J\left(\gamma_{t} ; u, \beta(u)\right)\left(\tau_{h}\right)$, we have $\xi\left(\tau_{-h}\right) \geq J\left(\gamma_{t} ; u, \beta(u)\right), \quad P$-a.s. for all $u \in \mathcal{U}_{t, T}$. So $\xi\left(\tau_{-h}\right) \geq I\left(\gamma_{t} ; \beta\right), P$-a.s., that is, $\xi \geq I\left(\gamma_{t} ; \beta\right)\left(\tau_{h}\right)$, $P$-a.s. Thus,

$$
\begin{aligned}
& J\left(\gamma_{t} ; u, \beta(u)\right)\left(\tau_{h}\right) \geq\left\{\operatorname{essup}_{u \in \mathscr{U}_{t, T}} J\left(\gamma_{t} ; u, \beta(u)\right)\right\}\left(\tau_{h}\right), \\
& P \text {-a.s., for any } u \in \mathscr{U}_{t, T} \text {. }
\end{aligned}
$$

Therefore,

$$
\begin{aligned}
\underset{u \in \mathscr{U}_{t, T}}{\operatorname{esssup}} & \left\{J\left(\gamma_{t} ; u, \beta(u)\right)\left(\tau_{h}\right)\right\} \\
\geq & \left\{\begin{array}{l}
\left.\operatorname{esssup}_{u \in \mathscr{U}_{t, T}} J\left(\gamma_{t} ; u, \beta(u)\right)\right\} \\
\left.\operatorname{eic}_{h}\right), \quad P \text {-a.s. }
\end{array}\right.
\end{aligned}
$$

From above we get

$$
\begin{aligned}
& \left\{\operatorname{esssup}_{u \in \mathscr{U}_{t, T}} J\left(\gamma_{t} ; u, \beta(u)\right)\right\}\left(\tau_{h}\right) \\
& =\underset{u \in \mathscr{U}_{t, T}}{\operatorname{esssup}}\left\{J\left(\gamma_{t} ; u, \beta(u)\right)\left(\tau_{h}\right)\right\}, \quad P \text {-a.s. }
\end{aligned}
$$

(4) Under the Girsanov transformation $\tau_{h}, W\left(\gamma_{t}\right)$ is invariant, that is,

$$
W\left(\gamma_{t}\right)\left(\tau_{h}\right)=W\left(\gamma_{t}\right), \quad P \text {-a.s., for any } h \in H \text {. }
$$

In fact, we can prove $\left\{\operatorname{essinf}_{\beta \in \mathscr{B}_{t, T}} I\left(\gamma_{t} ; \beta\right)\right\}\left(\tau_{h}\right)=$ $\operatorname{essinf}_{\beta \in \mathscr{B}_{t, T}}\left\{I\left(\gamma_{t} ; \beta\right)\left(\tau_{h}\right)\right\}$, P-a.s., for all $h \in H_{t}$, which is similar to the above step. From the above three steps, for all $h \in H_{t}$, we get

$$
\begin{aligned}
& W\left(\gamma_{t}\right)\left(\tau_{h}\right)=\left\{\begin{array}{ll}
\operatorname{essinf}_{\beta \in \mathscr{B}_{t, T}} & \operatorname{esssup} \\
u \in \mathscr{U}_{t, T}
\end{array}\left(\gamma_{t} ; u, \beta(u)\right)\right\}\left(\tau_{h}\right), \\
& =\underset{\beta \in \mathscr{B}_{t, T}}{\operatorname{essinf}} \operatorname{esssup}\left\{\mathscr{U}_{t, T}\left\{\left(\gamma_{t} ; u, \beta(u)\right)\left(\tau_{h}\right)\right\}\right. \text {, } \\
& =\underset{\beta \in \mathscr{B}_{t, T}}{\operatorname{essinf}} \operatorname{esssup}\left\{J\left(\mathscr{U}_{t, T} ; u\left(\tau_{h}\right), \beta^{h}\left(u\left(\tau_{h}\right)\right)\right)\right\}, \\
& =\underset{\beta \in \mathscr{B}_{t, T}}{\operatorname{essinf}} \underset{u \in \mathscr{U}_{t, T}}{\operatorname{esssup}} J\left(\gamma_{t} ; u, \beta(u)\right) \text {, } \\
& =W\left(\gamma_{t}\right), \quad P \text {-a.s.. }
\end{aligned}
$$

Note that $\left\{u\left(\tau_{h}\right) \mid u(\cdot) \in \mathscr{U}_{t, T}\right\}=\mathscr{U}_{t, T}$ and $\left\{\beta^{h} \mid \beta \epsilon\right.$ $\left.\mathscr{B}_{t, T}\right\}=\mathscr{B}_{t, T}$ have been used in the above equalities. So, for any $h \in H_{t}, W\left(\gamma_{t}\right)\left(\tau_{h}\right)=W\left(\gamma_{t}\right), P$-a.s. Thanks to $W\left(\gamma_{t}\right)$ is $\mathscr{F}_{t}$-measurable, this relation holds true for all $h \in H$. follows

To finish the proof, we also need the auxiliary lemma as

Lemma 13. Let $\zeta$ be a random variable defined over the classical Wiener space $\left(\Omega, \mathscr{F}_{T}, P\right)$, such that $\zeta\left(\tau_{h}\right)=\zeta, P$-a.s., for any $h \in H$. Then $\zeta=E \zeta, P$-a.s.

Proof. From Lemma 13 in Buckdahn and Li [10], we know for any $A \in \mathscr{B}(\mathbb{R}), \varphi \in L^{2}\left([0, T] ; \mathbb{R}^{d}\right)$,

$$
\begin{aligned}
& E\left[1_{\{\zeta \in A\}} \exp \left\{\int_{0}^{T} \varphi(s) d B(s)\right\}\right] \\
& =E\left[1_{\{\zeta \in A\}}\right] E\left[\exp \left\{\int_{0}^{T} \varphi(s) d B(s)\right\}\right] .
\end{aligned}
$$

For any $\varphi=\sum_{i=1}^{N} \varphi_{i} 1_{\left(t_{i-1}, t_{i}\right]}$, where $\varphi_{i} \in \mathbb{R}^{d}$, for $0 \leq i \leq N$ and $\left\{t_{i}\right\}_{i=0}^{N}$ is a finite partition of $[0, T]$, from (36),

$$
\begin{aligned}
& E\left[1_{\{\zeta \in A\}} \exp \left(\sum_{i=1}^{N} \varphi_{i} B\left(t_{i}\right)-B\left(t_{i-1}\right)\right)\right] \\
& =E\left[1_{\{\zeta \in A\}}\right] E\left[\exp \sum_{i=1}^{N} \varphi_{i}\left(B\left(t_{i}\right)-B\left(t_{i-1}\right)\right)\right] .
\end{aligned}
$$

Therefore, for any nonnegative integer $k_{i}$

$$
\begin{aligned}
& E\left[1_{\{\zeta \in A\}} \prod_{i=1}^{N}\left(B\left(t_{i}\right)-B\left(t_{i-1}\right)\right)^{k_{i}}\right] \\
& \quad=E\left[1_{\{\zeta \in A\}}\right] E\left[\prod_{i=1}^{N}\left(B\left(t_{i}\right)-B\left(t_{i-1}\right)\right)^{k_{i}}\right] .
\end{aligned}
$$

So, for any polynomial function $Q$, we have

$$
\begin{aligned}
E & {\left[1_{\{\zeta \in A\}} Q\left(B\left(t_{1}\right)-B\left(t_{0}\right), \ldots, B\left(t_{n}\right)-B\left(t_{n-1}\right)\right)\right] } \\
& =E\left[1_{\{\zeta \in A\}}\right] E\left[Q\left(B\left(t_{1}\right)-B\left(t_{0}\right), \ldots, B\left(t_{n}\right)-B\left(t_{n-1}\right)\right)\right] .
\end{aligned}
$$

Furthermore, for any $Q \in C_{b}\left(\mathbb{R}^{n}\right)$, we still have (39). Combining the arbitrariness of $A \in \mathscr{B}(\mathbb{R})$, we obtain $\zeta$ is independent of $\left(B\left(t_{1}\right)-B\left(t_{0}\right), \ldots, B\left(t_{n}\right)-B\left(t_{n-1}\right)\right)$, for all partition of $[0, T]$. Therefore, $\zeta$ is independent of $\mathscr{F}_{T}$ which implies $\zeta$ is independent of itself, that is $E \zeta=\zeta, P$-a.s.

In Ji and Yang [14], they proved the following estimates. 
Lemma 14. Under the assumption $(\mathrm{H})$, there exists some constant $C>0$ such that, for any $t \in[0, T], \gamma_{t}, \bar{\gamma}_{t} \in \Lambda, u(\cdot) \in$ $\mathcal{U}, v(\cdot) \in \mathscr{V}$,

$$
\begin{aligned}
& E\left[\sup _{s \in[t, T]}\left|X^{\gamma_{t}, u, v}(s)\right|^{2} \mid \mathscr{F}_{t}\right] \leq C\left(1+\left\|\gamma_{t}\right\|^{2}\right), \\
& E\left[\sup _{s \in[t, T]}\left|X^{\gamma_{t}, u, v}(s)-X^{\bar{\gamma}_{t}, u, v}(s)\right|^{2} \mid \mathscr{F}_{t}\right] \leq C\left\|\gamma_{t}-\bar{\gamma}_{t}\right\|^{2}, \\
& E\left[\sup _{s \in[t, T]}\left|Y^{\gamma_{t}, u, v}(s)\right|^{2}+\int_{t}^{T}\left|Z^{\gamma_{t}, u, v}(s)\right|^{2} d s \mid \mathscr{F}_{t}\right] \leq C\left(1+\left\|\gamma_{t}\right\|^{2}\right),
\end{aligned}
$$$$
E\left[\sup _{s \in[t, T]}\left|Y^{\gamma_{t}, u, v}(s)-Y^{\bar{\gamma}_{t}, u, v}(s)\right|^{2}\right.
$$$$
\left.+\int_{t}^{T}\left|Z^{\gamma_{t}, u, v}(s)-Z^{\bar{\gamma}_{t}, u, v}(s)\right|^{2} d s \mid \mathscr{F}_{t}\right] \leq C\left\|\gamma_{t}-\bar{\gamma}_{t}\right\|^{2} .
$$

From the definition of $W\left(\gamma_{t}\right)$ and Lemma 14, we have the following property.

Lemma 15. There exists some constant $C>0$ such that, for all $0 \leq t \leq T, \gamma_{t}, \bar{\gamma}_{t} \in \Lambda$,

$$
\begin{aligned}
& \text { (i) }\left|W\left(\gamma_{t}\right)-W\left(\bar{\gamma}_{t}\right)\right| \leq C\left\|\gamma_{t}-\bar{\gamma}_{t}\right\|, \\
& \text { (ii) }\left|W\left(\gamma_{t}\right)\right| \leq C\left(1+\left\|\gamma_{t}\right\|\right) .
\end{aligned}
$$

Now we adopt Peng's notion of stochastic backward semigroup (which was first introduced by Peng [9] to prove the DPP for stochastic control problems) to discuss a generalized DPP for our SDG (17), (22). First we define the family of backward semigroups associated with FBSDE (17).

For given $t \in[0, T], \gamma_{t} \in \Lambda$, a number $\delta \in(0, T-t]$, admissible control processes $u(\cdot) \in \mathcal{U}_{t, t+\delta}, v(\cdot) \in \mathscr{V}_{t, t+\delta}$, we set

$$
G_{s, t+\delta}^{\gamma_{t} ; u, v}[\eta]:=\widetilde{Y}^{\gamma_{t} ; u, v}(s), \quad s \in[t, t+\delta]
$$

where $\eta \in L^{2}\left(\Omega, \mathscr{F}_{t}^{t+\delta}, P ; \mathbb{R}\right),\left(\widetilde{Y}^{\gamma_{t} ; u, v}(s), \widetilde{Z}^{\gamma_{t} ; u, v}(s)\right)_{t \leq s \leq t+\delta}$ solves the following functional FBSDE on $[t, t+\delta]$ :

$$
\begin{aligned}
& d \widetilde{Y}^{\gamma_{t} ; u, v}(s) \\
& =-f\left(s, X_{s}^{\gamma_{t} ; u, v}, \widetilde{Y}^{\gamma_{t} ; u, v}(s), \widetilde{Z}^{\gamma_{t} ; u, v}(s), u(s), v(s)\right) d s \\
& +\widetilde{Z}^{\gamma_{t} ; u, v}(s) d B(s), \\
& \tilde{Y}^{\gamma_{t} ; u, v}(t+\delta)=\eta, \quad s \in[t, t+\delta] .
\end{aligned}
$$

Also, we have

$$
G_{t, T}^{\gamma_{t} ; u, v}\left[\Phi\left(X_{T}^{\gamma_{t} ; u, v}\right)\right]=G_{t, t+\delta}^{\gamma_{t} ; u, v}\left[Y^{\gamma_{t} ; u, v}(t+\delta)\right] .
$$

Theorem 16. Suppose $(\mathrm{H})$ holds true, the lower value function $W\left(\gamma_{t}\right)$ satisfies the following DPP: for any $t \in[0, T], \gamma_{t} \in \Lambda, \delta>$ 0 ,

$$
W\left(\gamma_{t}\right)=\underset{\beta \in \mathscr{B}_{t, t+\delta}}{\operatorname{essinf}} \underset{u \in \mathcal{U}_{t, t+\delta}}{\operatorname{esssup}} G_{t, t+\delta}^{\gamma_{t} ; u, \beta(u)}\left[W\left(X_{t+\delta}^{\gamma_{t} ; u, \beta(u)}\right)\right] .
$$

The proof is given in the appendix.

\section{Viscosity Solutions of Path-Dependent HJBI Equation}

Now we study the following path-dependent PDEs:

$$
\begin{gathered}
D_{t} W\left(\gamma_{t}\right)+H^{-}\left(\gamma_{t}, W, D_{x} W, D_{x x} W\right)=0, \\
W\left(\gamma_{T}\right)=\Phi\left(\gamma_{T}\right), \quad \gamma_{T} \in \Lambda \\
D_{t} U\left(\gamma_{t}\right)+H^{+}\left(\gamma_{t}, U, D_{x} U, D_{x x} U\right)=0, \\
U\left(\gamma_{t}\right)=\Phi\left(\gamma_{T}\right), \quad \gamma_{T} \in \Lambda,
\end{gathered}
$$

where

$$
\begin{aligned}
& H^{-}\left(\gamma_{t}, W, D_{x} W, D_{x x} W\right) \\
& =\sup _{u \in U} \inf _{v \in V} H\left(\gamma_{t}, W, D_{x} W, D_{x x} W, u, v\right), \\
& H^{+}\left(\gamma_{t}, U, D_{x} U, D_{x x} U\right) \\
& =\inf _{v \in V} \sup _{u \in U} H\left(\gamma_{t}, U, D_{x} U, D_{x x} U, u, v\right), \\
& H\left(\gamma_{t}, y, p, X, u, v\right) \\
& =\frac{1}{2} \operatorname{tr}\left(\sigma \sigma^{T}\left(\gamma_{t}, u, v\right) X\right)+p \cdot b\left(\gamma_{t}, u, v\right) \\
& \quad+f\left(\gamma_{t}, y, p \cdot \sigma\left(\gamma_{t}, u, v\right), u, v\right),
\end{aligned}
$$

where $\left(\gamma_{t}, y, p, X\right) \in \Lambda \times \mathbb{R} \times \mathbb{R}^{d} \times \mathbb{S}^{d}$ ( $\mathbb{S}^{d}$ denotes the set of $d \times d$ symmetric matrices).

We will show that the value function $W\left(\gamma_{t}\right)$ (resp., $U\left(\gamma_{t}\right)$ ) defined in (22) (resp., (23)) is a viscosity solution of the corresponding equation (46) (resp., (47)). First we give the definition of viscosity solution for this kind of PDEs. For more information on viscosity solution, the reader is referred to Crandall et al. [22].

Definition 17. A real-valued $\Lambda$-continuous function $W \in$ $\mathbb{C}(\Lambda)$ is called

(i) a viscosity subsolution of (46) if for any $\delta>0, \Gamma \in$ $\mathbb{C}_{l, b}^{1,2}(\Lambda), \gamma_{t} \in \Lambda$ satisfying $\Gamma \geq W$ on $\bigcup_{0 \leq s \leq \delta} \Lambda_{t+s}$ and $\Gamma\left(\gamma_{t}\right)=W\left(\gamma_{t}\right)$, one has

$$
D_{t} \Gamma\left(\gamma_{t}\right)+H^{-}\left(\gamma_{t}, \Gamma, D_{x} \Gamma, D_{x x} \Gamma\right) \geq 0,
$$

(ii) a viscosity supersolution of (46) if for any $\delta>0, \Gamma \epsilon$ $\mathbb{C}_{l, b}^{1,2}(\Lambda), \gamma_{t} \in \Lambda$ satisfying $\Gamma \leq W$ on $\bigcup_{0 \leq s \leq \delta} \Lambda_{t+s}$ and $\Gamma\left(\gamma_{t}\right)=W\left(\gamma_{t}\right)$, one has

$$
D_{t} \Gamma\left(\gamma_{t}\right)+H^{-}\left(\gamma_{t}, \Gamma, D_{x} \Gamma, D_{x x} \Gamma\right) \leq 0,
$$

(iii) a viscosity solution of (46) if it is both a viscosity suband supersolution of (46).

Theorem 18. Assume $(\mathrm{H})$ holds, the lower value function $W$ is a viscosity solution of path-dependent HJBI Equation (46) on $\Lambda$, the upper value function $U$ is a viscosity solution of (47). 
First we prove some helpful lemmas. For some fixed $\Gamma \in$ $\mathbb{C}_{l, b}^{1,2}(\Lambda)$, denote

$$
\begin{aligned}
F\left(\gamma_{s}, y, z, u, v\right) \\
=D_{s} \Gamma\left(\gamma_{s}\right)+\frac{1}{2} \operatorname{tr}\left(\sigma \sigma^{T}\left(\gamma_{s}, u, v\right) D_{x x} \Gamma\left(\gamma_{s}\right)\right) \\
\quad+D_{x} \Gamma\left(\gamma_{s}\right) \cdot b\left(\gamma_{s}, u, v\right) \\
\quad+f\left(\gamma_{s}, y+\Gamma\left(\gamma_{s}\right), z+D_{x} \Gamma\left(\gamma_{s}\right) \cdot \sigma\left(\gamma_{s}, u, v\right), u, v\right),
\end{aligned}
$$

where $\left(\gamma_{s}, y, z, u, v\right) \in \Lambda \times \mathbb{R} \times \mathbb{R}^{d} \times U \times V$.

Consider the following BSDE:

$$
\begin{aligned}
-d Y^{1, u, v}(s) & \\
= & F\left(X_{s}^{\gamma_{t} ; u, v}, Y^{1, u, v}(s), Z^{1, u, v}(s), u(s), v(s)\right) d s \\
- & Z^{1, u, v}(s) d B(s), \\
& Y^{1, u, v}(t+\delta)=0, \quad s \in[t, t+\delta] .
\end{aligned}
$$

Lemma 19. For every $s \in[t, t+\delta]$, one has the following:

$$
Y^{1, u, v}(s)=G_{s, t+\delta}^{\gamma_{t} ; u, v}\left[\Gamma\left(X_{t+\delta}^{\gamma_{t} ; u, v}\right)\right]-\Gamma\left(X_{s}^{\gamma_{t} ; u, v}\right), \quad P \text {-a.s. }
$$

Proof. Note $G_{s, t+\delta}^{\gamma_{t} ; u, v}\left[\Gamma\left(X_{t+\delta}^{\gamma_{t} ; u, v}\right)\right]$ is defined as $G_{s, t+\delta}^{\gamma_{t} ; u, v}\left[\Gamma\left(X_{t+\delta}^{\gamma_{t} ; u, v}\right)\right]=$ $Y^{u, v}(s)$ through the following BSDE:

$$
\begin{gathered}
-d Y^{u, v}(s)=f\left(X_{s}^{\gamma_{t} ; u, v}, Y^{u, v}(s), Z^{u, v}(s), u(s), v(s)\right) d s \\
-Z^{u, v}(s) d B(s), \\
Y^{u, v}(t+\delta)=\Gamma\left(X_{t+\delta}^{\gamma_{t} ; u, v}\right), \quad s \in[t, t+\delta] .
\end{gathered}
$$

Using Itô's formula to $\Gamma\left(X_{s}^{\gamma_{t} ; u, v}\right)$, we have

$$
d\left(Y^{u, v}(s)-\Gamma\left(X_{s}^{\gamma_{t} ; u, v}\right)\right)=d Y^{1, u, v}(s) .
$$

Combined with $Y^{u, v}(t+\delta)-\Gamma\left(X_{t+\delta}^{\gamma_{t} ; u, v}\right)=0=Y^{1, u, v}(t+\delta)$, we get the desired result.

Now consider the following BSDE:

$$
\begin{aligned}
&-d Y^{2, u, v}(s)=F\left(\gamma_{t}, Y^{2, u, v}(s), Z^{2, u, v}(s), u(s), v(s)\right) d s \\
&-Z^{2, u, v}(s) d B(s), \\
& Y^{2, u, v}(t+\delta)=0, \quad s \in[t, t+\delta] .
\end{aligned}
$$

Then, we have the following lemma.

Lemma 20. For every $u \in \mathcal{U}_{t, t+\delta}, v \in \mathscr{V}_{t, t+\delta}$, one has

$$
\left|Y^{1, u, v}(t)-Y^{2, u, v}(t)\right| \leq C \delta^{3 / 2}, \quad P \text {-a.s. }
$$

where $C$ is independent of the control processes $u, v$.
Proof. From Lemma 14, we know there exists some constant $C>0$ such that

$$
E\left[\sup _{s \in[t, T]}\left|X^{\gamma_{t} ; u, v}(s)\right|^{2} \mid \mathscr{F}_{t}\right] \leq C\left(1+\left\|\gamma_{t}\right\|^{2}\right)
$$

combined with

$$
\begin{aligned}
& E\left[\sup _{s \in[t, t+\delta]}\left|X^{\gamma_{t} ; u, v}(s)-\gamma_{t}(t)\right|^{2} \mid \mathscr{F}_{t}\right] \\
& \leq 2 E\left[\sup _{s \in[t, t+\delta]}\left|\int_{t}^{s} b\left(X_{r}^{\gamma_{t} ; u, v}, u(r), v(r)\right) d r\right|^{2} \mid \mathscr{F}_{t}\right] \\
& \quad+2 E\left[\sup _{s \in[t, t+\delta]}\left|\int_{t}^{s} \sigma\left(X_{r}^{\gamma_{t} ; u, v}, u(r), v(r)\right) d B(r)\right|^{2} \mid \mathscr{F}_{t}\right]
\end{aligned}
$$

we have

$$
E\left[\sup _{s \in[t, t+\delta]}\left|X^{\gamma_{t} ; u, v}(s)-\gamma_{t}(t)\right|^{2} \mid \mathscr{F}_{t}\right] \leq C \delta .
$$

From (52) and (54), using Lemma 8, set

$$
\begin{gathered}
\xi_{1}=\xi_{2}=0, \\
g(s, y, z)=F\left(X_{s}^{\gamma_{t} ; u, v}, y, z, u_{s}, v_{s}\right), \\
\varphi_{1}(s)=0, \\
\varphi_{2}(s)=F\left(\gamma_{t}, Y^{2, u, v}(s), Z^{2, u, v}(s), u_{s}, v_{s}\right) \\
-F\left(X_{s}^{\gamma_{t} ; u, v}, Y^{2, u, v}(s), Z^{2, u, v}(s), u_{s}, v_{s}\right) .
\end{gathered}
$$

Denote $\rho_{0}(r)=\left(1+|x|^{2}\right) r$, due to $b, \sigma, f$ are Lipishtiz and that they are of linear growth, $\Gamma \in \mathbb{C}_{b}^{1,2},\left|\varphi_{2}(s)\right| \leq \rho_{0}\left(\mid X_{s}^{\gamma_{t} ; u, v}-\right.$ $\left.\gamma_{t}(t) \mid\right)$, we have

$$
\begin{aligned}
& E\left[\int _ { t } ^ { t + \delta } \left(\left|Y^{1, u, v}(s)-Y^{2, u, v}(s)\right|^{2}\right.\right. \\
& \left.\left.\quad+\left|Z^{1, u, v}(s)-Z^{2, u, v}(s)\right|^{2}\right) d s \mid \mathscr{F}_{t}\right] \\
& \quad \leq E\left[\int_{t}^{t+\delta} \rho_{0}^{2}\left(\left|X_{s}^{\gamma_{t} ; u, v}-\gamma_{t}(t)\right|\right) d s \mid \mathscr{F}_{t}\right] \\
& \leq C \delta E\left[\sup _{s \in[t, t+\delta]} \rho_{0}^{2}\left(\left|X_{s}^{\gamma_{t} ; u, v}-\gamma_{t}(t)\right|\right) \mid \mathscr{F}_{t}\right] \\
& \leq C \delta^{2} .
\end{aligned}
$$


So,

$$
\begin{aligned}
& \left|Y^{1, u, v}(t)-Y^{2, u, v}(t)\right| \\
& =\left|E\left[\left(Y^{1, u, v}(s)-Y^{2, u, v}(s)\right) \mid \mathscr{F}_{t}\right]\right| \\
& =\mid E\left[\int _ { t } ^ { t + \delta } \left(F\left(X_{s}^{\gamma_{t} ; u, v}, Y^{1, u, v}(s), Z^{1, u, v}(s), u(s), v(s)\right)\right.\right. \\
& \left.\left.-F\left(\gamma_{t}, Y^{2, u, v}(s), Z^{2, u, v}(s), u(s), v(s)\right)\right) d s \mid \mathscr{F}_{t}\right] \mid \\
& \leq C E\left[\int _ { t } ^ { t + \delta } \left[\rho_{0}\left(\left|X_{s}^{\gamma_{t} ; u, v}-\gamma_{t}\right|\right)+\left|Y^{1, u, v}(s)-Y^{2, u, v}(s)\right|\right.\right. \\
& \left.\left.+\left|Z^{1, u, v}(s)-Z^{2, u, v}(s)\right|\right] d s \mid \mathscr{F}_{t}\right] \\
& \leq C E\left[\int_{t}^{t+\delta} \rho_{0}\left(\left|X_{s}^{\gamma_{t} ; u, v}-\gamma_{t}\right|\right) d s \mid \mathscr{F}_{t}\right] \\
& +C \delta^{1 / 2} E\left[\int_{t}^{t+\delta}\left|Y^{1, u, v}(s)-Y^{2, u, v}(s)\right|^{2} d s \mid \mathscr{F}_{t}\right]^{1 / 2} \\
& +C \delta^{1 / 2} E\left[\int_{t}^{t+\delta}\left|Z^{1, u, v}(s)-Z^{2, u, v}(s)\right|^{2} d s \mid \mathscr{F}_{t}\right]^{1 / 2} \\
& \leq C \delta^{3 / 2}
\end{aligned}
$$

Lemma 21. Denote $Y_{0}(\cdot)$ by the solution of the following ordinary differential equation:

$$
\begin{gathered}
-d Y_{0}(s)=F_{0}\left(\gamma_{t}, Y_{0}(s), 0\right) d s, \quad s \in[t, t+\delta], \\
Y_{0}(t+\delta)=0,
\end{gathered}
$$

where $F_{0}\left(\gamma_{t}, y, z\right):=\sup _{u \in U} \inf _{v \in V} F\left(\gamma_{t}, y, z, u, v\right)$. Then, P-a.s.,

$$
Y_{0}(t)=\underset{u \in \mathscr{U}_{t, t+\delta}}{\operatorname{esssup}} \underset{v \in \mathscr{V}_{t, t+\delta}}{\operatorname{essinf}} Y^{2, u, v}(t)
$$

Proof. First we define a function as follows:

$$
\begin{array}{r}
F_{1}\left(\gamma_{t}, y, z, u\right):=\inf _{v \in V} F\left(\gamma_{t}, y, z, u, v\right), \\
\left(\gamma_{t}, y, z, u\right) \in \Lambda \times \mathbb{R} \times \mathbb{R}^{d} \times U .
\end{array}
$$

Consider the following equation:

$$
\begin{gathered}
-d Y^{3, u}(s)=F_{1}\left(\gamma_{t}, Y^{3, u}(s), Z^{3, u}(s), u(s)\right) d s \\
-Z^{3, u}(s) d B(s), \quad s \in[t, t+\delta], \\
Y^{3, u}(t+\delta)=0,
\end{gathered}
$$

according to Lemma 6 , for every $u \in \mathcal{U}_{t, t+\delta}$, there exists a unique $\left(Y^{3, u}, Z^{3, u}\right)$ solving (68). Also,

$$
Y^{3, u}(t)=\underset{v \in \mathscr{V}_{t, t+\delta}}{\operatorname{essinf}} Y^{2, u, v}(t), \quad P \text {-a.s. for every } u \in \mathcal{U}_{t, t+\delta} \text {. }
$$

In fact, according to the definition of $F_{1}$ and Lemma 7, we have

$$
\begin{array}{r}
Y^{3, u}(t) \leq Y^{2, u, v}(t), \quad P \text {-a.s. for any } v \in \mathscr{V}_{t, t+\delta} \\
\text { for every } u \in \mathscr{U}_{t, t+\delta} .
\end{array}
$$

On the other side, we have the existence of a measurable function $v^{4}: \mathbb{R} \times \mathbb{R}^{d} \times U \rightarrow V$ such that

$$
\begin{aligned}
& F_{1}\left(\gamma_{t}, y, z, u\right) \\
& \quad=F\left(\gamma_{t}, y, z, u, v^{4}(y, z, u)\right), \quad \text { for any } y, z, u .
\end{aligned}
$$

Set $\widetilde{v}^{4}(s)=v^{4}\left(Y^{3, u}(s), Z^{3, u}(s), u(s)\right), s \in[t, t+\delta]$, we know $\widetilde{v}^{4} \in \mathscr{V}_{t, t+\delta}$. Therefore, $\left(Y^{3, u}, Z^{3, u}\right)=\left(Y^{2, u, \widetilde{v}^{4}}, Z^{2, u, \widetilde{v}^{4}}\right)$ which is due to the uniqueness of the solution of (68). In particular, $Y^{3, u}(t)=Y^{2, u, \widetilde{v}^{4}}(t), P$-a.s. for every $u \in \mathcal{U}_{t, t+\delta}$. Hence, $Y^{3, u}(t)=\operatorname{essinf}_{v \in \mathscr{V}_{t, t+\delta}} Y^{2, u, v}(t), P$-a.s. for every $u \in \mathscr{U}_{t, t+\delta}$.

Similarly, from $F_{0}\left(\gamma_{t}, y, z\right)=\sup _{u \in U} F_{1}\left(\gamma_{t}, y, z, u\right)$, we also derive

$$
Y_{0}(t)=\operatorname{esssup}_{u \in \mathscr{U}_{t, t+\delta}} Y^{3, u}(t)=\operatorname{esssup}_{u \in \mathscr{U}_{t, t+\delta}} \underset{v \in \mathscr{V}_{t, t+\delta}}{\operatorname{essinf}} Y^{2, u, v}(t), \quad P \text {-a.s. }
$$

Lemma 22. For every $u \in \mathcal{U}_{t, t+\delta}, v \in \mathscr{V}_{t, t+\delta}$, one has

$E\left[\int_{t}^{t+\delta}\left(\left|Y^{2, u, v}(s)\right|+\left|Z^{2, u, v}(s)\right|\right) d s \mid \mathscr{F}_{t}\right] \leq C \delta^{3 / 2}, \quad P$-a.s.,

where the constant $C$ is independent of the control processes $u, v$.

Proof. Due to $F\left(\gamma_{t}, \cdot, \cdot, u, v\right)$ is linear growth in $(y, z)$, uniformly in $(u, v)$, from Lemma 8 , we know there exists a constant $C>0$ which does not depend on $\delta$ nor on the controls $u$ and $v$, such that, $P$-a.s.

$$
\begin{gathered}
\left|Y^{2, u, v}(s)\right|^{2} \leq C \delta \\
E\left[\int_{s}^{t+\delta}\left|Z^{2, u, v}(r)\right|^{2} d r \mid \mathscr{F}_{s}\right] \leq C \delta .
\end{gathered}
$$


Moreover, from (54), we have

$$
\begin{aligned}
& \left|Y^{2, u, v}(s)\right| \\
& \quad \leq E\left[\int_{s}^{t+\delta} F\left(\gamma_{t}, Y^{2, u, v}(r), Z^{2, u, v}(r), u(r), v(r)\right) d r \mid \mathscr{F}_{s}\right] \\
& \quad \leq C E\left[\int_{s}^{t+\delta}\left(1+\left\|\gamma_{t}\right\|^{2}+\left|Y^{2, u, v}(r)\right|+\left|Z^{2, u, v}(r)\right|\right) d r \mid \mathscr{F}_{s}\right] \\
& \quad \leq C \delta+C \delta^{1 / 2} E\left[\int_{s}^{t+\delta}\left|Z^{2, u, v}(r)\right|^{2} d r \mid \mathscr{F}_{s}\right] \\
& \quad \leq C \delta, \quad s \in[t, t+\delta],
\end{aligned}
$$

and applying Itô's formula, we get $E\left[\int_{t}^{t+\delta}\left|Z^{2, u, v}(s)\right|^{2} d s \quad \mid\right.$ $\left.\mathscr{F}_{t}\right] \leq C \delta^{2}, P$-a.s. Therefore,

$$
\begin{aligned}
& E\left[\int_{t}^{t+\delta}\left(\left|Y^{2, u, v}(s)\right|+\left|Z^{2, u, v}(s)\right|\right) d s \mid \mathscr{F}_{t}\right] \\
& \quad \leq C \delta^{2}+C \delta^{1 / 2}\left(E\left[\int_{t}^{t+\delta}\left|Z^{2, u, v}(s)\right|^{2} d r \mid \mathscr{F}_{t}\right]\right)^{1 / 2} \\
& \quad \leq C \delta^{3 / 2}, \quad P \text {-a.s. }
\end{aligned}
$$

Now we continue the proof of Theorem 18.

Proof. (1) First we will prove $W\left(\gamma_{t}\right)$ is a viscosity supersolution. Given $\gamma_{t} \in \Lambda$, for any $\delta>0$, suppose $\Gamma\left(\gamma_{t}\right)=W\left(\gamma_{t}\right)$ and $W \geq \Gamma$ on $\bigcup_{0 \leq s \leq \delta} \Lambda_{t+s}$.

According to the DPP (Theorem 16), we have

$$
\Gamma\left(\gamma_{t}\right)=W\left(\gamma_{t}\right)=\underset{\beta \in \mathscr{B}_{t, t+\delta}}{\operatorname{essinf}} \underset{u \in \mathcal{U}_{t, t+\delta}}{\operatorname{esssup}} G_{t, t+\delta}^{\gamma_{t} u, \beta(u)}\left[W\left(X_{t+\delta}^{\gamma_{t} ; u, \beta(u)}\right)\right] .
$$

From $W \geq \Gamma$ on $\bigcup_{0 \leq s \leq \delta} \Lambda_{t+s}$ as well as the monotonicity property of $G_{t, t+\delta}^{\gamma_{t} ; u, \beta(u)}[\cdot]$ (Lemma 7) we have

$$
\underset{\beta \in \mathscr{B}_{t, t+\delta}}{\operatorname{essinf}} \operatorname{esssup} G_{u \in \mathscr{U}_{t, t+\delta}}^{\gamma_{t} ; u, \beta(u)}\left[\Gamma\left(X_{t+\delta}^{\gamma_{t} ; u, \beta(u)}\right)\right]-\Gamma\left(\gamma_{t}\right) \leq 0 .
$$

By Lemma 19, we have

$$
\underset{\beta \in \mathscr{\mathscr { S }}_{t, t+\delta}}{\operatorname{essinf}} \underset{u \in \mathscr{U}_{t, t+\delta}}{\operatorname{esssup}} Y^{1, u, \beta(u)}(t) \leq 0, \quad P \text {-a.s. }
$$

Thus, from Lemma 20,

$$
\underset{\beta \in \mathscr{B}_{t, t+\delta}}{\operatorname{essinf}} \underset{u \in \mathscr{U}_{t, t+\delta}}{\operatorname{esssup}} Y^{2, u, \beta(u)}(t) \leq C \delta^{3 / 2}, \quad P \text {-a.s. }
$$

Therefore, from essinf ${ }_{v \in \mathscr{V}_{t, t+\delta}} Y^{2, u, v}(t) \leq Y^{2, u, \beta(u)}(t), \beta \in$ $\mathscr{B}_{t, t+\delta}$, we have

$$
\begin{aligned}
& \underset{u \in \mathscr{U}_{t, t+\delta}}{\operatorname{esssup}} \underset{v \in \mathscr{V}_{t, t+\delta}}{\operatorname{essinf}} Y^{2, u, v}(t) \\
& \quad \leq \underset{\beta \in \mathscr{B}_{t, t+\delta}}{\operatorname{essinf}} \operatorname{esssup} \\
& u \in \mathscr{U}_{t, t+\delta}
\end{aligned}
$$

Thus, by Lemma $21, Y_{0}(t) \leq C \delta^{3 / 2}, P$-a.s., where $Y_{0}(\cdot)$ solves the ODE (65). Consequently,

$$
\begin{aligned}
C \delta^{1 / 2} \geq & \frac{1}{\delta} Y_{0}(t)=\frac{1}{\delta} \int_{t}^{t+\delta} F_{0}\left(\gamma_{t}, Y_{0}(s), 0\right) d s, \quad \delta>0, \\
& \sup _{u \in U} \inf _{v \in V} F\left(\gamma_{t}, 0,0, u, v\right)=F_{0}\left(\gamma_{t}, 0,0\right) \leq 0 .
\end{aligned}
$$

Obviously, according to the definition of $F, W$ is a viscosity supersolution of (46).

(2) Now we prove $W$ is a viscosity subsolution.

Given $\gamma_{t} \in \Lambda$, for any $\delta>0$, suppose $\Gamma\left(\gamma_{t}\right)=W\left(\gamma_{t}\right)$ and $\Gamma \geq W$ on $\bigcup_{0 \leq s \leq \delta} \Lambda_{t+s}$.

We need to prove

$$
\sup _{u \in \mathscr{U}} \inf _{v \in V} F\left(\gamma_{t}, 0,0, u, v\right)=F_{0}\left(\gamma_{t}, 0,0\right) \geq 0 \text {. }
$$

Suppose it does not hold true. So we have some $\theta>0$ such that

$$
F_{0}\left(\gamma_{t}, 0,0\right)=\sup _{u \in \mathscr{U}} \inf _{v \in V} F\left(\gamma_{t}, 0,0, u, v\right) \leq-\theta<0,
$$

and there exists a measurable function $\psi: U \rightarrow V$ such that

$$
F\left(\gamma_{t}, 0,0, u, \psi(u)\right) \leq-\frac{3}{4} \theta, \quad \forall u \in U
$$

Moreover, from the DPP (Theorem 16),

$$
\Gamma\left(\gamma_{t}\right)=W\left(\gamma_{t}\right)=\underset{\beta \in \mathscr{B}_{t, t+\delta}}{\operatorname{essinf}} \underset{u \in \mathscr{U}_{t, t+\delta}}{\operatorname{esssup}} G_{t, t+\delta}^{\gamma_{t} ; u, \beta(u)}\left[W\left(X_{t+\delta}^{\gamma_{t}, u, \beta(u)}\right)\right] .
$$

Again from $W \leq \Gamma$ on $\bigcup_{0 \leq s \leq \delta} \Lambda_{t+s}$ as well as the monotonicity property of $G_{t, t+\delta}^{\gamma_{t} ; u, \beta(u)}[\cdot]$ (Lemma 7) we get

$$
\underset{\beta \in \mathscr{B}_{t, t+\delta}}{\operatorname{essinf}} \operatorname{esssup} G_{u \in \mathscr{U}_{t, t+\delta}}^{\gamma_{t} ; u, \beta(u)}\left[\Gamma\left(X_{t+\delta}^{u, \beta(u)}\right)\right]-\Gamma\left(\gamma_{t}\right) \geq 0, \quad P \text {-a.s. }
$$

Then, similar to (1), from the definition of backward semigroup, we have

$$
\underset{\beta \in \mathscr{B}_{t, t+\delta}}{\operatorname{essinf}} \underset{u \in \mathscr{U}_{t, t+\delta}}{\operatorname{esssup}} Y^{1, u, \beta(u)}(t) \geq 0, \quad P \text {-a.s., }
$$

in particular,

$$
\operatorname{esssup}_{u \in \mathcal{U}_{t, t+\delta}} Y^{1, u, \psi(u)}(t) \geq 0, \quad P \text {-a.s. }
$$

Setting $\psi_{s}(u)(\omega)=\psi(u(s)(\omega)),(s, \omega) \in[t, T] \times \Omega, \psi$ can be regarded as an element of $\mathscr{B}_{t, t+\delta}$. Given any $\varepsilon>0$ we can select $u^{\varepsilon} \in \mathcal{U}_{t, t+\delta}$ satisfying $Y^{1, u^{\varepsilon}, \psi\left(u^{\varepsilon}\right)}(t) \geq-\varepsilon \delta$.

From Lemma 20 we have

$$
Y^{2, u^{\varepsilon}, \psi\left(u^{\varepsilon}\right)}(t) \geq-C \delta^{3 / 2}-\varepsilon \delta, \quad P \text {-a.s. }
$$


Moreover, from (54) we have

$$
\begin{aligned}
& Y^{2, u^{\varepsilon}, \psi\left(u^{\varepsilon}\right)}(t) \\
& =E\left[\int _ { t } ^ { t + \delta } F \left(\gamma_{t}, Y^{2, u^{\varepsilon}, \psi\left(u^{\varepsilon}\right)}(s), Z^{2, u^{\varepsilon}, \psi\left(u^{\varepsilon}\right)}(s),\right.\right. \\
& \left.\left.u^{\varepsilon}(s), \psi\left(u^{\varepsilon}(s)\right)\right) d s \mid \mathscr{F}_{t}\right] \\
& \leq E\left[\int _ { t } ^ { t + \delta } \left(C\left|Y^{2, u^{\varepsilon}, \psi\left(u^{\varepsilon}\right)}(s)\right|+C\left|Z^{2, u^{\varepsilon}, \psi\left(u^{\varepsilon}\right)}(s)\right|\right.\right. \\
& \left.\left.+F\left(\gamma_{t}, 0,0, u^{\varepsilon}(s), \psi\left(u^{\varepsilon}(s)\right)\right)\right) d s \mid \mathscr{F}_{t}\right] \\
& \leq C \delta^{3 / 2}-\frac{3}{4} \theta \delta, \quad P \text {-a.s. }
\end{aligned}
$$

From (91) and (92), we have $-C \delta^{1 / 2}-\varepsilon \leq C \delta^{1 / 2}-$ $(3 / 4) \theta, \quad P$-a.s. Letting $\delta \downarrow 0$, and then $\varepsilon \downarrow 0$, we get $\theta \leq 0$, which produces a contradiction. Consequently,

$$
\sup _{u \in U} \inf _{v \in V} F\left(\gamma_{t}, 0,0, u, v\right)=F_{0}\left(\gamma_{t}, 0,0\right) \leq 0 .
$$

According to the definition of $F, W$ is a viscosity supersolution of (46). At last, from the above two steps, we derive that $W$ is a viscosity solution of (46).

The similar argument for $U$, we also get that $U$ is a viscosity solution of (47).

\section{Appendix}

\section{Proof of Theorem 16 (DPP)}

Proof. For convenience, we set

$$
W_{\delta}\left(\gamma_{t}\right)=\underset{\beta \in \mathscr{B}_{t, t+\delta}}{\operatorname{essinf}} \operatorname{esssup} G_{u \in \mathscr{U}_{t, t+\delta}}^{\gamma_{t} ; u, \beta(u)}\left[W\left(X_{t+\delta}^{\gamma_{t} ; u, \beta(u)}\right)\right] .
$$

We want to prove $W_{\delta}\left(\gamma_{t}\right)$ and $W\left(\gamma_{t}\right)$ coincide. For it we only need to prove the following three lemmas.

Lemma A.1. $W_{\delta}\left(\gamma_{t}\right)$ is deterministic.

The proof of this lemma is similar to the proof of Proposition 12 , so we omit it here.

Lemma A.2. $W_{\delta}\left(\gamma_{t}\right) \leq W\left(\gamma_{t}\right)$.

Proof. For any fixed $\beta \in \mathscr{B}_{t, T}$, given a $u_{2}(\cdot) \in \mathcal{U}_{t+\delta, T}$, the restriction $\beta_{1}$ of $\beta$ to $\mathscr{U}_{t, t+\delta}$ can be defined as follows:

$$
\beta_{1}\left(u_{1}\right):=\left.\beta\left(u_{1} \oplus u_{2}\right)\right|_{[t, t+\delta]}, \quad u_{1}(\cdot) \in \mathcal{U}_{t, t+\delta},
$$

where $u_{1} \oplus u_{2}:=u_{1} \mathbf{1}_{[t, t+\delta]}+u_{2} \mathbf{1}_{(t+\delta, T]}$, generalizes $u_{1}(\cdot)$ to an element of $\mathscr{U}_{t, T}$. Clearly, $\beta_{1} \in \mathscr{B}_{t, t+\delta}$. Also, due to the nonanticipativity property of $\beta$ we know $\beta_{1}$ is free of the choice of $u_{2}(\cdot) \in \mathcal{U}_{t+\delta, T}$. Therefore, due to the definition of $W_{\delta}\left(\gamma_{t}\right)$

$$
W_{\delta}\left(\gamma_{t}\right) \leq \operatorname{esssup}_{u_{1} \in \mathscr{U}_{t, t+\delta}} G_{t, t+\delta}^{\gamma_{t} ; u_{1}, \beta_{1}\left(u_{1}\right)}\left[W\left(X_{t+\delta}^{\gamma_{t} ; u_{1}, \beta_{1}\left(u_{1}\right)}\right)\right], \quad P \text {-a.s. }
$$

We put $I_{\delta}\left(\gamma_{t}, u, v\right):=G_{t, t+\delta}^{\gamma_{t} ; u, v}\left[W\left(X_{t+\delta}^{\gamma_{t} ; u, v}\right)\right]$. Then there exists a sequence $\left\{u_{i}^{1}, i \geq \mathbf{1}\right\} \subset \mathcal{U}_{t, t+\delta}$, such that

$$
\begin{aligned}
I_{\delta}\left(\gamma_{t}, \beta_{1}\right) & :=\operatorname{esssup}_{u_{1} \in \mathscr{U}_{t, t+\delta}} I_{\delta}\left(\gamma_{t} ; u_{1}, \beta_{1}\left(u_{1}\right)\right) \\
& =\sup _{i \geq 1} I_{\delta}\left(\gamma_{t} ; u_{i}^{1}, \beta_{1}\left(u_{i}^{1}\right)\right), \quad P \text {-a.s. }
\end{aligned}
$$

For any $\varepsilon>0$, we set $\widetilde{\Gamma}_{i}:=\left\{I_{\delta}\left(\gamma_{t}, \beta_{1}\right) \leq I_{\delta}\left(\gamma_{t} ; u_{i}^{1}, \beta_{1}\left(u_{i}^{1}\right)\right)+\right.$ $\varepsilon\} \in \mathscr{F}_{t}, i \geq 1$. Then the sets $\Gamma_{1}:=\widetilde{\Gamma}_{1}, \Gamma_{i}:=\widetilde{\Gamma}_{i} \mid$ $\left(\bigcup_{l=1}^{i-1} \widetilde{\Gamma}_{l}\right) \in \mathscr{F}_{t}, \quad i \geq 2$, form an $\left(\Omega, \mathscr{F}_{t}\right)$-partition, Thus, $u_{1}^{\varepsilon}:=\sum_{i \geq 1} \mathbf{1}_{\Gamma_{i}} u_{i}^{1} \in \mathcal{U}_{t, t+\delta}$. Also, from the nonanticipativity of $\beta_{1}$ we have $\beta_{1}\left(u_{1}^{\varepsilon}\right)=\sum_{i \geq 1} \mathbf{1}_{\Gamma_{i}} \beta_{1}\left(u_{i}^{1}\right)$, and due to the uniqueness of the solution of the functional FBSDE, we have $I_{\delta}\left(\gamma_{t} ; u_{1}^{\varepsilon}, \beta_{1}\left(u_{1}^{\varepsilon}\right)\right)=\sum_{i \geq 1} \mathbf{1}_{\Gamma_{i}} I_{\delta}\left(\gamma_{t} ; u_{i}^{1}, \beta_{1}\left(u_{i}^{1}\right)\right), P$-a.s. So,

$$
\begin{aligned}
W_{\delta}\left(\gamma_{t}\right) \leq I_{\delta}\left(\gamma_{t} ; \beta_{1}\right) & \leq \sum_{i \geq 1} \mathbf{1}_{\Gamma_{i}} I_{\delta}\left(\gamma_{t} ; u_{i}^{1}, \beta_{1}\left(u_{i}^{1}\right)\right)+\varepsilon \\
& =I_{\delta}\left(\gamma_{t} ; u_{1}^{\varepsilon}, \beta_{1}\left(u_{1}^{\varepsilon}\right)\right)+\varepsilon \\
& =G_{t, t+\delta}^{\gamma_{t} ; u_{1}^{\varepsilon}, \beta_{1}\left(u_{1}^{\varepsilon}\right)}\left[W\left(X_{t+\delta}^{\gamma_{t} ; u_{1}^{\varepsilon}, \beta_{1}\left(u_{1}^{\varepsilon}\right)}\right)\right]+\varepsilon .
\end{aligned}
$$

On the other hand, since $\beta_{1}(\cdot):=\beta\left(\cdot \oplus u_{2}\right) \in \mathscr{B}_{t, t+\delta}$ is independent of $u_{2}(\cdot) \in \mathcal{U}_{t+\delta, T}$, we define $\beta_{2}\left(u_{2}\right):=\beta\left(u_{1}^{\varepsilon} \oplus\right.$ $\left.u_{2}\right)\left.\right|_{[t+\delta, T]}$, for all $u_{2}(\cdot) \in \mathscr{U}_{t+\delta, T}$. According to the definition of $W\left(\gamma_{t}\right)$ we get, for any $y \in \mathbb{R}^{n}$,

$$
W\left(\gamma_{t}\right) \leq \operatorname{esssup}_{u_{2} \in \mathscr{U}_{t+\delta, T}} J\left(\gamma_{t} ; u_{2}, \beta_{2}\left(u_{2}\right)\right), \quad P \text {-a.s. }
$$

Recalling that there exists a constant $C \in \mathbb{R}$ such that

(i) $\left|W\left(\gamma_{t}\right)-W\left(\bar{\gamma}_{t}\right)\right| \leq C\left\|\gamma_{t}-\bar{\gamma}_{t}\right\| \quad$ for any $\gamma_{t}, \bar{\gamma}_{t} \in \Lambda$,

(ii) $\left|J\left(\gamma_{t} ; u_{2}, \beta_{2}\left(u_{2}\right)\right)-J\left(\bar{\gamma}_{t} ; u_{2}, \beta_{2}\left(u_{2}\right)\right)\right|$

$$
\leq C\left\|\gamma_{t}-\bar{\gamma}_{t}\right\|, \quad P \text {-a.s. for any } u_{2} \in \mathcal{U}_{t+\delta, T} .
$$

We can show by approximating $X_{t+\delta}^{\gamma_{t} ; u_{1}^{\varepsilon}, \beta_{1}\left(u_{1}^{\varepsilon}\right)}$ that

$$
\begin{aligned}
& W\left(X_{t+\delta}^{\gamma_{t} ; u_{1}^{\varepsilon}, \beta_{1}\left(u_{1}^{\varepsilon}\right)}\right) \\
& \quad \leq \operatorname{esssup}_{u_{2} \in \mathcal{U}_{t+\delta, T}} J\left(X_{t+\delta}^{\gamma_{t} ; u_{1}^{\varepsilon}, \beta_{1}\left(u_{1}^{\varepsilon}\right)} ; u_{2}, \beta_{2}\left(u_{2}\right)\right), \quad P \text {-a.s. }
\end{aligned}
$$


Now we estimate the right side of the latter inequality, there exists some sequence $\left\{u_{j}^{2}, j \geq 1\right\} \subset \mathcal{U}_{t+\delta, T}$ such that

$$
\begin{aligned}
& \underset{u_{2} \in \mathscr{U}_{t+\delta, T}}{\operatorname{esssup}} J\left(X_{t+\delta}^{\gamma_{t} ; u_{1}^{\varepsilon}, \beta_{1}\left(u_{1}^{\varepsilon}\right)} ; u_{2}, \beta_{2}\left(u_{2}\right)\right) \\
& \quad=\sup _{j \geq 1} J\left(X_{t+\delta}^{\gamma_{t} ; u_{1}^{\varepsilon}, \beta_{1}\left(u_{1}^{\varepsilon}\right)} ; u_{j}^{2}, \beta_{2}\left(u_{j}^{2}\right)\right), \quad P \text {-a.s. }
\end{aligned}
$$

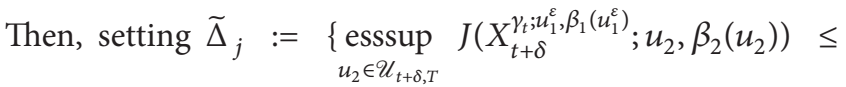
$\left.J\left(X_{t+\delta}^{\gamma_{t} ; u_{1}^{\varepsilon}, \beta_{1}\left(u_{1}^{\varepsilon}\right)} ; u_{j}^{2}, \beta_{2}\left(u_{j}^{2}\right)\right)+\varepsilon\right\} \in \mathscr{F}_{t+\delta}, j \geq 1$; we have the sets $\Delta_{1}:=\widetilde{\Delta}_{1}, \quad \Delta_{j}:=\widetilde{\Delta}_{j} \backslash\left(\bigcup_{l=1}^{j-1} \widetilde{\Delta}_{l}\right) \in \mathscr{F}_{t+\delta}, j \geq 2$ forms an $\left(\Omega, \mathscr{F}_{t+\delta}\right)$-partition and $u_{2}^{\varepsilon}:=\sum_{j \geq 1} \mathbf{1}_{\Delta_{j}} u_{j}^{2} \in \mathcal{u}_{t+\delta, T}$. Due to the nonanticipativity of $\beta_{2}$ we get $\beta_{2}\left(u_{2}^{\varepsilon}\right)=\sum_{j \geq 1} \mathbf{1}_{\Delta_{j}} \beta_{2}\left(u_{j}^{2}\right)$, also from the definitions of $\beta_{1}, \beta_{2}$, we have $\beta\left(u_{1}^{\varepsilon} \oplus u_{2}^{\varepsilon}\right)=$ $\beta_{1}\left(u_{1}^{\varepsilon}\right) \oplus \beta_{2}\left(u_{2}^{\varepsilon}\right)$. Therefore, due to the uniqueness of the solution of functional FBSDE, we get

$$
\begin{aligned}
& J\left(X_{t+\delta}^{\gamma_{t} ; u_{1}^{\varepsilon}, \beta_{1}\left(u_{1}^{\varepsilon}\right)} ; u_{2}^{\varepsilon}, \beta_{2}\left(u_{2}^{\varepsilon}\right)\right) \\
& =Y^{X_{t+\delta}^{\gamma_{t} ; u_{1}^{\varepsilon}, \beta_{1}\left(u_{1}^{\varepsilon}\right)} ; u_{2}^{\varepsilon}, \beta_{2}\left(u_{2}^{\varepsilon}\right)}(t+\delta) \\
& =\sum_{j \geq 1} \mathbf{1}_{\Delta_{j}} Y^{X_{t+\delta}^{\gamma_{t} ; u_{1}^{\varepsilon}, \beta_{1}\left(u_{1}^{\varepsilon}\right)} ; u_{j}^{2}, \beta_{2}\left(u_{j}^{2}\right)}(t+\delta) \\
& =\sum_{j \geq 1} \mathbf{1}_{\Delta_{j}} J\left(X_{t+\delta}^{\gamma_{t} ; u_{1}^{\varepsilon}, \beta_{1}\left(u_{1}^{\varepsilon}\right)} ; u_{j}^{2}, \beta_{2}\left(u_{j}^{2}\right)\right), \quad P \text {-a.s. }
\end{aligned}
$$

So,

$$
\begin{aligned}
& W\left(X_{t+\delta}^{\left.\gamma_{t} ; u_{1}^{\varepsilon}, \beta_{1}, u_{1}^{\varepsilon}\right)}\right) \\
& \quad \leq \operatorname{esssup}_{u_{2} \in \mathscr{U}_{t+\delta, T}} J\left(X_{t+\delta}^{\gamma_{t} ; u_{1}^{\varepsilon}, \beta_{1}\left(u_{1}^{\varepsilon}\right)} ; u_{2}, \beta_{2}\left(u_{2}\right)\right) \\
& \quad \leq \sum_{j \geq 1} \mathbf{1}_{\Delta_{j}} J\left(X_{t+\delta}^{\gamma_{t} ; u_{1}^{\varepsilon}, \beta_{1}\left(u_{1}^{\varepsilon}\right)} ; u_{1}^{\varepsilon} \oplus u_{j}^{2}, \beta\left(u_{1}^{\varepsilon} \oplus u_{j}^{2}\right)\right)+\varepsilon \\
& \quad=J\left(X_{t+\delta}^{\gamma_{t}, u_{1}^{\varepsilon}, \beta_{1}\left(u_{1}^{\varepsilon}\right)} ; u_{1}^{\varepsilon} \oplus u_{2}^{\varepsilon}, \beta\left(u_{1}^{\varepsilon} \oplus u_{2}^{\varepsilon}\right)\right)+\varepsilon \\
& \quad=J\left(X_{t+\delta}^{\gamma_{t} ; u_{1}^{\varepsilon}, \beta_{1}\left(u_{1}^{\varepsilon}\right)} ; u^{\varepsilon}, \beta\left(u^{\varepsilon}\right)\right)+\varepsilon, P \text {-a.s., }
\end{aligned}
$$

where $u^{\varepsilon}:=u_{1}^{\varepsilon} \oplus u_{2}^{\varepsilon} \in \mathscr{u}_{t, T}$. From (A.5), (A.11) and the comparison theorem for BSDEs, we know

$$
\begin{aligned}
W_{\delta} & \left(\gamma_{t}\right) \\
& \leq G_{t, t+\delta}^{\gamma_{t}, u_{1}^{\varepsilon}, \beta_{1}\left(u_{1}^{\varepsilon}\right)}\left[J\left(X_{t+\delta}^{\gamma_{t} ; u_{1}^{\varepsilon}, \beta_{1}\left(u_{1}^{\varepsilon}\right)} ; u^{\varepsilon}, \beta\left(u^{\varepsilon}\right)\right)+\varepsilon\right]+\varepsilon \\
& \leq G_{t, t+\delta}^{\gamma_{t} ; u_{1}^{\varepsilon}, \beta_{1}\left(u_{1}^{\varepsilon}\right)}\left[J\left(X_{t+\delta}^{\gamma_{t} ; u_{1}^{\varepsilon}, \beta_{1}\left(u_{1}^{\varepsilon}\right)} ; u^{\varepsilon}, \beta\left(u^{\varepsilon}\right)\right)\right]+(C+1) \varepsilon \\
& =G_{t, t+\delta}^{\gamma_{t} ; u^{\varepsilon}, \beta\left(u^{\varepsilon}\right)}\left[J\left(X_{t+\delta}^{\gamma_{t} ; u_{1}^{\varepsilon}, \beta_{1}\left(u_{1}^{\varepsilon}\right)} ; u^{\varepsilon}, \beta\left(u^{\varepsilon}\right)\right)\right]+(C+1) \varepsilon \\
& =J\left(\gamma_{t} ; u^{\varepsilon}, \beta\left(u^{\varepsilon}\right)\right)+(C+1) \varepsilon \\
& =Y^{\gamma_{t} ; u^{\varepsilon}, \beta\left(u^{\varepsilon}\right)}(t)+(C+1) \varepsilon, \\
& \leq \underset{u \in \mathscr{U}_{t, T}}{\operatorname{esssup} Y^{\gamma_{t}} ; u, \beta(u)}(t)+(C+1) \varepsilon, \quad P \text {-a.s. }
\end{aligned}
$$

Because of the arbitrariness of $\beta \in \mathscr{B}_{t, T}$, (A.12) holds true for all $\beta \in \mathscr{B}_{t, T}$. Thus,

$$
\begin{aligned}
W_{\delta}\left(\gamma_{t}\right) & \leq \underset{\beta \in \mathscr{B}_{t, T}}{\operatorname{essinf}} \operatorname{esssup} Y_{u \in \mathscr{U}_{t, T}}^{\gamma_{t} ; u, \beta(u)}(t)+(C+1) \varepsilon \\
& =W\left(\gamma_{t}\right)+(C+1) \varepsilon .
\end{aligned}
$$

Therefore, letting $\varepsilon \downarrow 0$, we have $W_{\delta}\left(\gamma_{t}\right) \leq W\left(\gamma_{t}\right)$.

Lemma A.3. $W\left(\gamma_{t}\right) \leq W_{\delta}\left(\gamma_{t}\right)$

Proof. We keep the notations in the above lemma. From the definition of $W_{\delta}\left(\gamma_{t}\right)$ we know

$$
\begin{aligned}
W_{\delta}\left(\gamma_{t}\right) & =\underset{\beta_{1} \in \mathscr{B}_{t, t+\delta}}{\operatorname{essinf}} \operatorname{esssup} G_{1} \in \mathscr{U}_{t, t+\delta}^{\gamma_{t} ; u_{1}, \beta_{1}\left(u_{1}\right)}\left[W\left(X_{t+\delta}^{\gamma_{t} ; u_{1}, \beta_{1}\left(u_{1}\right)}\right)\right] \\
& =\underset{\beta_{1} \in \mathscr{B}_{t, t+\delta}}{\operatorname{essinf}} I_{\delta}\left(\gamma_{t}, \beta_{1}\right),
\end{aligned}
$$

and the existence of some sequence $\left\{\beta_{i}^{1}, i \geq 1\right\} \subset \mathscr{B}_{t, t+\delta}$ such that

$$
W_{\delta}\left(\gamma_{t}\right)=\inf _{i \geq 1} I_{\delta}\left(\gamma_{t}, \beta_{i}^{1}\right), \quad P \text {-a.s. }
$$

For any $\varepsilon>0$, we set $\widetilde{\Pi}_{i}:=\left\{I_{\delta}\left(\gamma_{t}, \beta_{i}^{1}\right)-\varepsilon \leq W_{\delta}\left(\gamma_{t}\right)\right\} \in$ $\mathscr{F}_{t}, \quad i \geq 1, \Pi_{1}:=\widetilde{\Pi}_{1}$ and $\Pi_{i}:=\widetilde{\Pi}_{i} \backslash\left(\bigcup_{l=1}^{i-1} \widetilde{\Pi}_{l}\right) \in \mathscr{F}_{t}, i \geq$ 2. Then, $\left\{\Pi_{i}, i \geq 1\right\}$ forms an $\left(\Omega, \mathscr{F}_{t}\right)$-partition, $\beta_{1}^{\varepsilon}:=$ $\sum_{i \geq 1} \mathbf{1}_{\Lambda_{i}} \beta_{i}^{1} \in \mathscr{B}_{t, t+\delta}$, combining the uniqueness of the solution of the functional FBSDE, we derive $I_{\delta}\left(\gamma_{t}, u_{1}, \beta_{1}^{\varepsilon}\left(u_{1}\right)\right)=$ $\sum_{i \geq 1} \mathbf{1}_{\Lambda_{i}} I_{\delta}\left(\gamma_{t}, u_{1}, \beta_{i}^{1}\left(u_{1}\right)\right), P$-a.s., for all $u_{1}(\cdot) \in \mathscr{u}_{t, t+\delta}$.

$$
\begin{aligned}
W_{\delta}\left(\gamma_{t}\right) \geq & \sum_{i \geq 1} \mathbf{1}_{\Pi_{i}} I_{\delta}\left(\gamma_{t}, \beta_{i}^{1}\right)-\varepsilon \\
\geq & \sum_{i \geq 1} \mathbf{1}_{\Pi_{i}} I_{\delta}\left(\gamma_{t}, u_{1}, \beta_{i}^{1}\left(u_{1}\right)\right)-\varepsilon \\
= & I_{\delta}\left(\gamma_{t}, u_{1}, \beta_{1}^{\varepsilon}\left(u_{1}\right)\right)-\varepsilon \\
= & G_{t, t+\delta}^{\gamma_{t}, u_{1}, \beta_{1}^{\varepsilon}\left(u_{1}\right)}\left[W\left(X_{t+\delta}^{\gamma_{t} ; u_{1}, \beta_{1}^{\varepsilon}\left(u_{1}\right)}\right)\right]-\varepsilon, \\
& P \text {-a.s., } \forall u_{1} \in \mathscr{U}_{t, t+\delta} .
\end{aligned}
$$


On the other hand, according to the definition of $W\left(\gamma_{t}\right)$, we deduce that, for any $x \in C\left([0, t+\delta] ; \mathbb{R}^{n}\right)$, there exists $\beta^{x, \varepsilon} \in$ $\mathscr{B}_{t+\delta, T}$ such that

$$
W\left(x_{t+\delta}\right) \geq \underset{u_{2} \in \mathcal{U}_{t+\delta, T}}{\operatorname{esssup}} J\left(x_{t+\delta} ; u_{2}, \beta^{x, \varepsilon}\left(u_{2}\right)\right)-\varepsilon, \quad P \text {-a.s. }
$$

Let $\left\{O_{i}\right\}_{i \geq 1}$ be a Borel partition of $C\left([0, t+\delta] ; \mathbb{R}^{n}\right)$ and $\operatorname{diam}\left(O_{i}\right) \leq \varepsilon, i \geq 1$. Let $\gamma^{i}$ be an arbitrarily fixed element of $O_{i}, i \geq 1$. Defining $\left[X_{t+\delta}^{\gamma_{t} ; u_{1}, \beta_{1}^{\varepsilon}\left(u_{1}\right)}\right]:=\sum_{i \geq 1} \gamma_{t+\delta}^{i} \mathbf{1}_{\left\{X_{t+\delta}^{\gamma_{t} ; u_{1}, \beta_{1}^{\varepsilon}\left(u_{1}\right)} \in O_{i}\right\}}$, we have

$$
\left|X_{t+\delta}^{\gamma_{t} ; u_{1}, \beta_{1}^{\varepsilon}\left(u_{1}\right)}-\left[X_{t+\delta}^{\gamma_{t} ; u_{1}, \beta_{1}^{\varepsilon}\left(u_{1}\right)}\right]\right| \leq \varepsilon
$$

everywhere on $\Omega, \forall u_{1} \in \mathcal{U}_{t, t+\delta}$.

And, for every $\gamma^{i}$, there exists some $\beta^{\gamma^{i}, \varepsilon} \in \mathscr{B}_{t+\delta, T}$ satisfying (A.17), and, $\beta_{u_{1}}^{\varepsilon}:=\sum_{i \geq 1} \mathbf{1}_{\left\{X_{t+\delta}^{\gamma_{t} ; u_{1}, \beta_{1}^{\varepsilon}\left(u_{1}\right)} \in O_{i}\right\}} \beta^{\gamma^{i}, \varepsilon} \in \mathscr{B}_{t+\delta, T}$.

Next we define the new $\beta^{\varepsilon}(u):=\beta_{1}^{\varepsilon}\left(u_{1}\right) \oplus \beta_{u_{1}}^{\varepsilon}\left(u_{2}\right), u \in$ $\mathcal{u}_{t, T}$, where $u_{1}=\left.u\right|_{[t, t+\delta]}, u_{2}=\left.u\right|_{(t+\delta, T]}$ (which are the restrictions of $u$ to $[t, t+\delta] \times \Omega$ and $(t+\delta, T] \times \Omega$, resp.). Clearly, $\beta^{\varepsilon}: \mathscr{U}_{t, T} \rightarrow \mathscr{V}_{t, T}$. And $\beta^{\varepsilon}$ is nonanticipating. In fact, let $S: \Omega \rightarrow[t, T]$ be an $\mathbb{F}$-stopping time and $u, u^{\prime} \in \mathcal{U}_{t, T}$ be such that $u \equiv u^{\prime}$ on $[[t, S]]$. Decomposing $u, u^{\prime}$ into $u_{1}, u_{1}^{\prime} \in$ $\mathcal{u}_{t, t+\delta}, u_{2}, u_{2}^{\prime} \in \mathcal{u}_{t+\delta, T}$ such that $u=u_{1} \oplus u_{1}^{\prime}$ and $u=u_{2} \oplus u_{2}^{\prime}$. From $u_{1} \equiv u_{1}^{\prime}$ on $[[t, S \wedge(t+\delta)]]$, we get $\beta_{1}^{\varepsilon}\left(u_{1}\right)=\beta_{1}^{\varepsilon}\left(u_{1}^{\prime}\right)$ on $[[t, S \wedge(t+\delta)]]$ (note that $\beta_{1}^{\varepsilon}$ is nonanticipating). On the other side, from $u_{2} \equiv u_{2}^{\prime}$ on $[[t+\delta, S \vee(t+\delta)]](\subset(t+\delta, T] \times\{S>$ $t+\delta\})$, and on $\{S>t+\delta\}$ we get $X_{t+\delta}^{\gamma_{t} ; u_{1}, \beta_{1}^{\varepsilon}\left(u_{1}\right)}=X_{t+\delta}^{\gamma_{t} ; u_{1}^{\prime}, \beta_{1}^{\varepsilon}\left(u_{1}^{\prime}\right)}$. Thus, from the definition, $\beta_{u_{1}}^{\varepsilon}=\beta_{u_{1}^{\prime}}^{\varepsilon}$ on $\{S>t+\delta\}$ and $\beta_{u_{1}}^{\varepsilon}\left(u_{2}\right)=\beta_{u_{1}^{\prime}}^{\varepsilon}\left(u_{2}^{\prime}\right)$ on] $\left.\left.]+\delta, S \vee(t+\delta)\right]\right]$. This produces $\beta^{\varepsilon}(u)=\beta_{1}^{\varepsilon}\left(u_{1}\right) \oplus \beta_{u_{1}}^{\varepsilon}\left(u_{2}\right)=\beta_{1}^{\varepsilon}\left(u_{1}^{\prime}\right) \oplus \beta_{u_{1}^{\prime}}^{\varepsilon}\left(u_{2}^{\prime}\right)$ on $[[t, S]]$, from which we know $\beta^{\varepsilon} \in \mathscr{B}_{t, T}$.

For an arbitrarily chosen $u \in \mathscr{U}_{t, T}$, decompose it into $u_{1}=$ $\left.u\right|_{(t, t+\delta]} \in \mathcal{U}_{t, t+\delta}$, and $u_{2}=\left.u\right|_{[t+\delta, T]} \in \mathcal{u}_{t+\delta, T}$. From (A.16), (A.7)-(i), (A.18), and the comparison theorem, we get

$$
\begin{aligned}
& W_{\delta}\left(\gamma_{t}\right) \geq G_{t, t+\delta}^{\gamma_{t} ; u_{1}, \beta_{1}^{\varepsilon}\left(u_{1}\right)}\left[W\left(X_{t+\delta}^{\gamma_{t} ; u_{1}, \beta_{1}^{\varepsilon}\left(u_{1}\right)}\right)\right]-\varepsilon \\
& \geq G_{t, t+\delta}^{\gamma_{t} ; u_{1}, \beta_{1}^{\varepsilon}\left(u_{1}\right)}\left[W\left(\left[X_{t+\delta}^{\gamma_{t} ; u_{1}, \beta_{1}^{\varepsilon}\left(u_{1}\right)}\right]\right)-C \varepsilon\right]-\varepsilon \\
& \geq G_{t, t+\delta}^{\gamma_{t} ; u_{1}, \beta_{1}^{\varepsilon}\left(u_{1}\right)}\left[W\left(\left[X_{t+\delta}^{\gamma_{t} ; u_{1}, \beta_{1}^{\varepsilon}\left(u_{1}\right)}\right]\right)\right]-C \varepsilon \\
& =G_{t, t+\delta}^{\gamma_{t} ; u_{1}, \beta_{1}^{\varepsilon}\left(u_{1}\right)}\left[\sum_{i \geq 1} 1_{\left\{X_{t+\delta}^{\gamma_{t} ; u_{1}, \beta_{1}^{\varepsilon}\left(u_{1}\right)} \in O_{i}\right\}} W\left(\gamma_{t+\delta}^{i}\right)\right]-C \varepsilon,
\end{aligned}
$$

Moreover, from (A.19), (A.7)-(ii), (A.17), and the comparison theorem, we have

$$
\begin{aligned}
& W_{\delta}\left(\gamma_{t}\right) \\
& \geq G_{t, t+\delta}^{\gamma_{t} ; u_{1}, \beta_{1}^{\varepsilon}\left(u_{1}\right)}\left[\sum_{i \geq 1} \mathbf{1}_{\left\{X_{t+\delta}^{\gamma_{t} ; u_{1}, \beta_{1}^{\varepsilon}\left(u_{1}\right)} \in O_{i}\right\}} J\left(\gamma_{t+\delta}^{i} ; u_{2}, \beta^{\gamma^{i}, \varepsilon}\left(u_{2}\right)\right)-\varepsilon\right]-C \varepsilon \\
& \geq G_{t, t+\delta}^{\gamma_{t} ; u_{1}, \beta_{1}^{\varepsilon}\left(u_{1}\right)}\left[\sum_{i \geq 1} \mathbf{1}_{\left\{X_{t+\delta}^{\gamma_{t} ; u_{1}, \beta_{1}^{\varepsilon}\left(u_{1}\right)} \in O_{i}\right\}} J\left(\gamma_{t+\delta}^{i} ; u_{2}, \beta^{\gamma^{i}, \varepsilon}\left(u_{2}\right)\right)\right]-C \varepsilon \\
& \quad=G_{t, t+\delta}^{\gamma_{t} ; u_{1}, \beta_{1}^{\varepsilon}\left(u_{1}\right)}\left[J\left(\left[X_{t+\delta}^{\gamma_{t} ; u_{1}, \beta_{1}^{\varepsilon}\left(u_{1}\right)}\right] ; u_{2}, \beta_{u_{1}}^{\varepsilon}\left(u_{2}\right)\right)\right]-C \varepsilon \\
& \quad \geq G_{t, t+\delta}^{\gamma_{t} ; u_{1}, \beta_{1}^{\varepsilon}\left(u_{1}\right)}\left[J\left(X_{t+\delta}^{\gamma_{t} ; u_{1}, \beta_{1}^{\varepsilon}\left(u_{1}\right)} ; u_{2}, \beta_{u_{1}}^{\varepsilon}\left(u_{2}\right)\right)-C \varepsilon\right]-C \varepsilon \\
& \geq G_{t, t+\delta}^{\gamma_{t} ; u_{1}, \beta_{1}^{\varepsilon}\left(u_{1}\right)}\left[J\left(X_{t+\delta}^{\gamma_{t} ; u_{1}, \beta_{1}^{\varepsilon}\left(u_{1}\right)} ; u_{2}, \beta_{u_{1}}^{\varepsilon}\left(u_{2}\right)\right)\right]-C \varepsilon \\
& \quad=G_{t, t+\delta}^{\gamma_{t} ; u^{\varepsilon}, \beta^{\varepsilon}(u)}\left[Y^{\gamma_{t} ; u, \beta^{\varepsilon}(u)}(t+\delta)\right]-C \varepsilon \\
& \quad=Y^{\gamma_{t} ; u_{1}, \beta^{\varepsilon}(u)}(t)-C \varepsilon, \quad P-\text { a.s., for any } u \in \mathscr{U}_{t, T} .
\end{aligned}
$$

Therefore,

$$
\begin{array}{rl}
W_{\delta}\left(\gamma_{t}\right) & \geq \underset{u \in \mathscr{U}_{t, T}}{\operatorname{esssup}} J\left(\gamma_{t} ; u, \beta^{\varepsilon}(u)\right)-C \varepsilon \\
& \geq \underset{\beta \in \mathscr{B}_{t, T}}{\operatorname{essinf}} \operatorname{esssup} \\
u \in \mathscr{U}_{t, T} & J\left(\gamma_{t} ; u, \beta(u)\right)-C \varepsilon \\
& =W\left(\gamma_{t}\right)-C \varepsilon, \quad P \text {-a.s. }
\end{array}
$$

Letting $\varepsilon \downarrow 0$ we derive $W_{\delta}\left(\gamma_{t}\right) \geq W\left(\gamma_{t}\right)$.

Remark A.4. (a) (i) For each $\beta \in \mathscr{B}_{t, t+\delta}$, there exists some $u^{\varepsilon}(\cdot) \in U_{t, t+\delta}$ such that

$$
\begin{array}{r}
W\left(\gamma_{t}\right)\left(=W_{\delta}\left(\gamma_{t}\right)\right) \leq G_{t, t+\delta}^{\gamma_{t} ; u^{\varepsilon}, \beta\left(u^{\varepsilon}\right)}\left[W\left(X_{t+\delta}^{\gamma_{t} ; u^{\varepsilon}, \beta\left(u^{\varepsilon}\right)}\right)\right]+\varepsilon \\
P \text {-a.s. }
\end{array}
$$
$U_{t, t+\delta}$

(ii) There exists some $\beta^{\varepsilon}(\cdot) \in B_{t, t+\delta}$ such that, for all $u(\cdot) \epsilon$

$$
\begin{array}{r}
W\left(\gamma_{t}\right)\left(=W_{\delta}\left(\gamma_{t}\right)\right) \geq G_{t, t+\delta}^{\gamma_{t} ; u^{\varepsilon}, \beta^{\varepsilon}(u)}\left[W\left(X_{t+\delta}^{\gamma_{t} ; u, \beta^{\varepsilon}(u)}\right)\right]-\varepsilon, \\
P \text {-a.s. }
\end{array}
$$

(b) From Proposition 12, we know the lower value function $W$ is deterministic. So, by choosing $\delta=T-t$ and taking expectation on both sides of (A.22), (A.23), we get $W\left(\gamma_{t}\right)=$ $\inf _{\beta \in \mathscr{B}_{t, T}} \sup _{u \in \mathscr{U}_{t, T}} E\left[J\left(\gamma_{t} ; u, \beta(u)\right)\right]$.

\section{Acknowledgments}

This work was supported by National Natural Science Foundation of China (no. 11171187, no. 10871118, and no. 10921101); 
the Programme of Introducing Talents of Discipline to Universities of China (no. B12023), and Program for New Century Excellent Talents in University of China.

\section{References}

[1] E. Pardoux and S. G. Peng, "Adapted solution of a backward stochastic differential equation," Systems \& Control Letters, vol. 14, no. 1, pp. 55-61, 1990.

[2] S. G. Peng, "A general stochastic maximum principle for optimal control problems," SIAM Journal on Control and Optimization, vol. 28, no. 4, pp. 966-979, 1990.

[3] S. Hamadene and J.-P. Lepeltier, "Zero-sum stochastic differential games and backward equations," Systems \& Control Letters, vol. 24, no. 4, pp. 259-263, 1995.

[4] S. Hamadene, J.-P. Lepeltier, and S. G. Peng, "BSDEs with continuous coefficients and stochastic differential games," in Backward Stochastic Differential Equations, vol. 364 of Pitman Research Notes in Math Series, pp. 115-128, El Karoui Mazliak, 1997.

[5] N. El Karoui, S. Peng, and M. C. Quenez, "Backward stochastic differential equations in finance," Mathematical Finance, vol. 7, no. 1, pp. 1-71, 1997.

[6] S. G. Peng, "Probabilistic interpretation for systems of quasilinear parabolic partial differential equations," Stochastics and Stochastics Reports, vol. 37, no. 1-2, pp. 61-74, 1991.

[7] S. G. Peng, "A generalized dynamic programming principle and Hamilton-Jacobi-Bellman equation," Stochastics and Stochastics Reports, vol. 38, no. 2, pp. 119-134, 1992.

[8] D. Duffie and L. G. Epstein, "Stochastic differential utility," Econometrica, vol. 60, no. 2, pp. 353-394, 1992.

[9] S. G. Peng, "Backward stochastic differential equationsstochastic optimization theory and viscosity solutions of HJB equations," in Topics on Stochastic Analysis, J. A. Yan, S. G. Peng, S. Z. Fang, and L. M. Wu, Eds., pp. 85-138, Science Press, Beijing, China, 1997.

[10] R. Buckdahn and J. Li, "Stochastic differential games and viscosity solutions of Hamilton-Jacobi-Bellman-Isaacs equations," SIAM Journal on Control and Optimization, vol. 47, no. 1, pp. 444-475, 2008.

[11] W. H. Fleming and H. M. Soner, Controlled Markov Processes and Viscosity Solutions, vol. 25, Springer, New York, NY, USA, Second edition, 2006.

[12] Z. Wu and Z. Y. Yu, "Dynamic programming principle for one kind of stochastic recursive optimal control problem and Hamilton-Jacobi-Bellman equation," SIAM Journal on Control and Optimization, vol. 47, no. 5, pp. 2616-2641, 2008.

[13] J. Yong and X. Y. Zhou, Stochastic Controls: Hamiltonian Systems and HJB Equations, vol. 43, Springer, New York, NY, USA, 1999.

[14] S. L. Ji and S. Z. Yang, "An optimal control problem for functional forward-backward stochastic systems and related Path-dependent HJB equations," http://arxiv.org/abs/1204.6543

[15] B. Dupire, "Functional Itô's Calculus," Portfolio Research Paper, Bloomberg, 2009.

[16] R. Cont and D. A. Fournié, "A functional extension of the Itô's formula," Comptes Rendus Mathématique, vol. 348, no. 1-2, pp. 57-61, 2010.

[17] R. Cont and D. A. Fournié, "Change of variable formulas for non-anticipative functionals on path space," Journal of Functional Analysis, vol. 259, no. 4, pp. 1043-1072, 2010.
[18] R. Cont and D. A. Fournié, "Functional Itô's calculus and stochastic integral representation of martingales," The Annals of Probability, vol. 41, no. 1, pp. 109-133, 2013.

[19] S. G. Peng and F. L. Wang, "BSDE, path-dependent PDE and nonlinear Feynman-Kac formula," In press, http://arxiv.org/ abs/1108.4317.

[20] I. Ekren, C. Keller, N. Touzi, and J. Zhang, "On viscosity solutions of path dependent PDEs," Annals of Probability. In press, http://arxiv.org/abs/1109.5971 .

[21] S. G. Peng, "Note on viscosity solution of path-dependent PDE and G-Martingales," Probability. In press, http://arxiv .org/abs/1106.1144.

[22] M. G. Crandall, H. Ishii, and P.-L. Lions, "User's guide to viscosity solutions of second order partial differential equations," Bulletin of the American Mathematical Society, vol. 27, no. 1, pp. 1-67, 1992. 


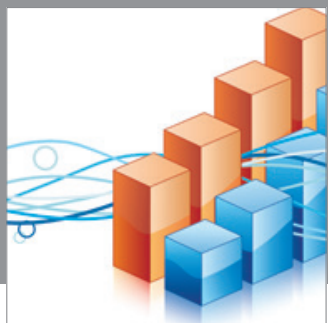

Advances in

Operations Research

mansans

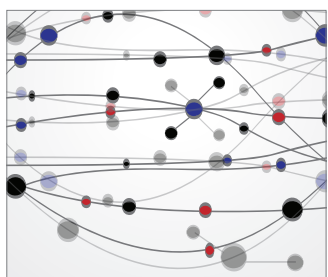

The Scientific World Journal
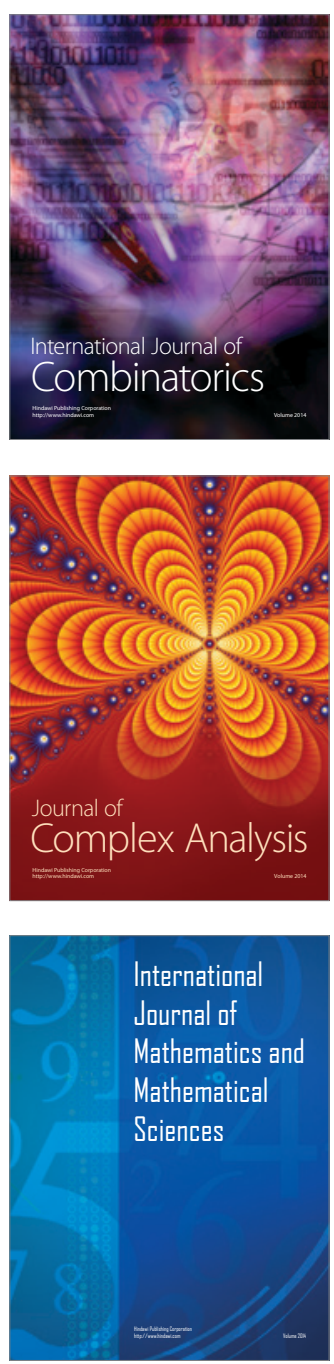
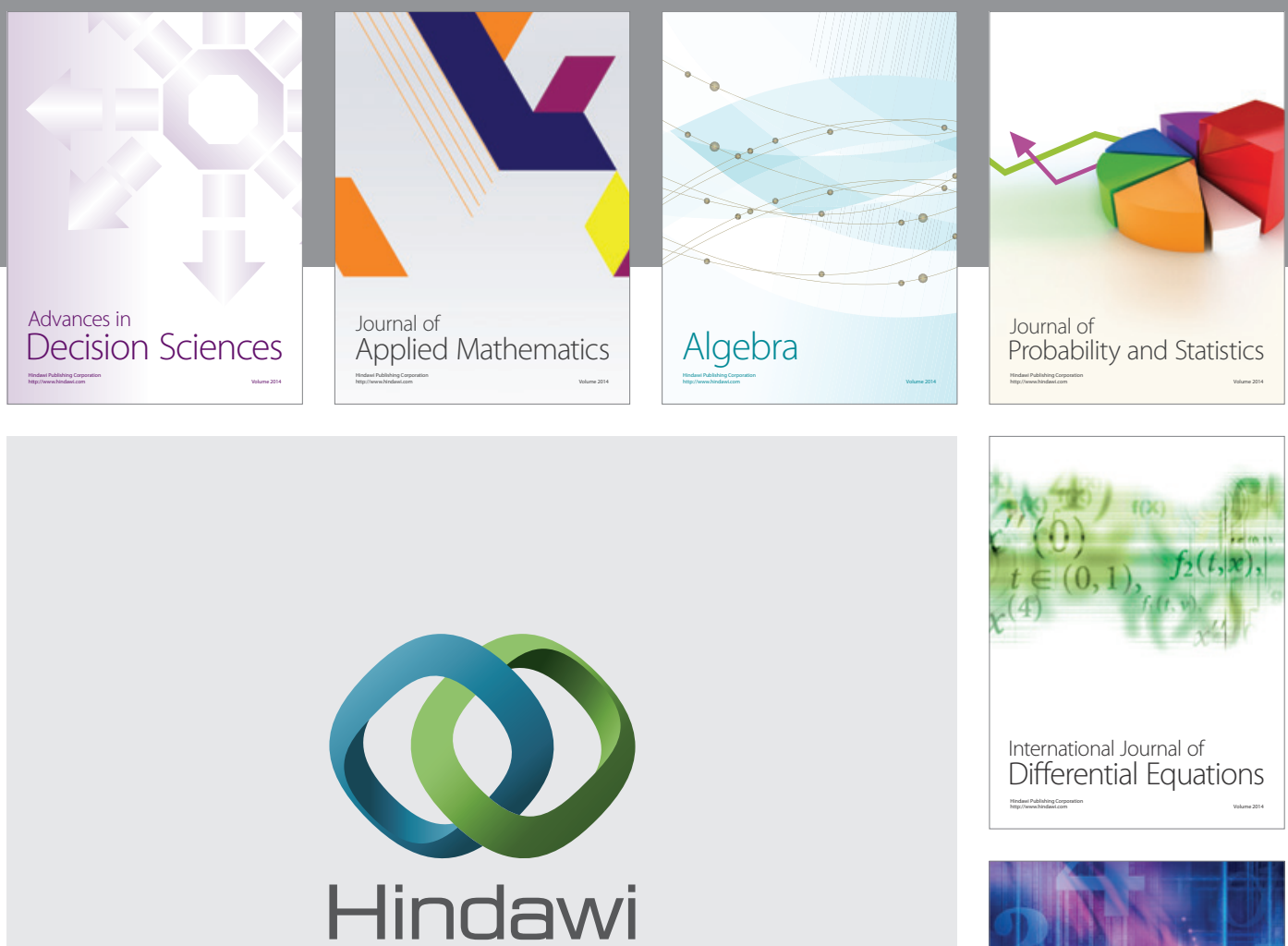

Submit your manuscripts at http://www.hindawi.com
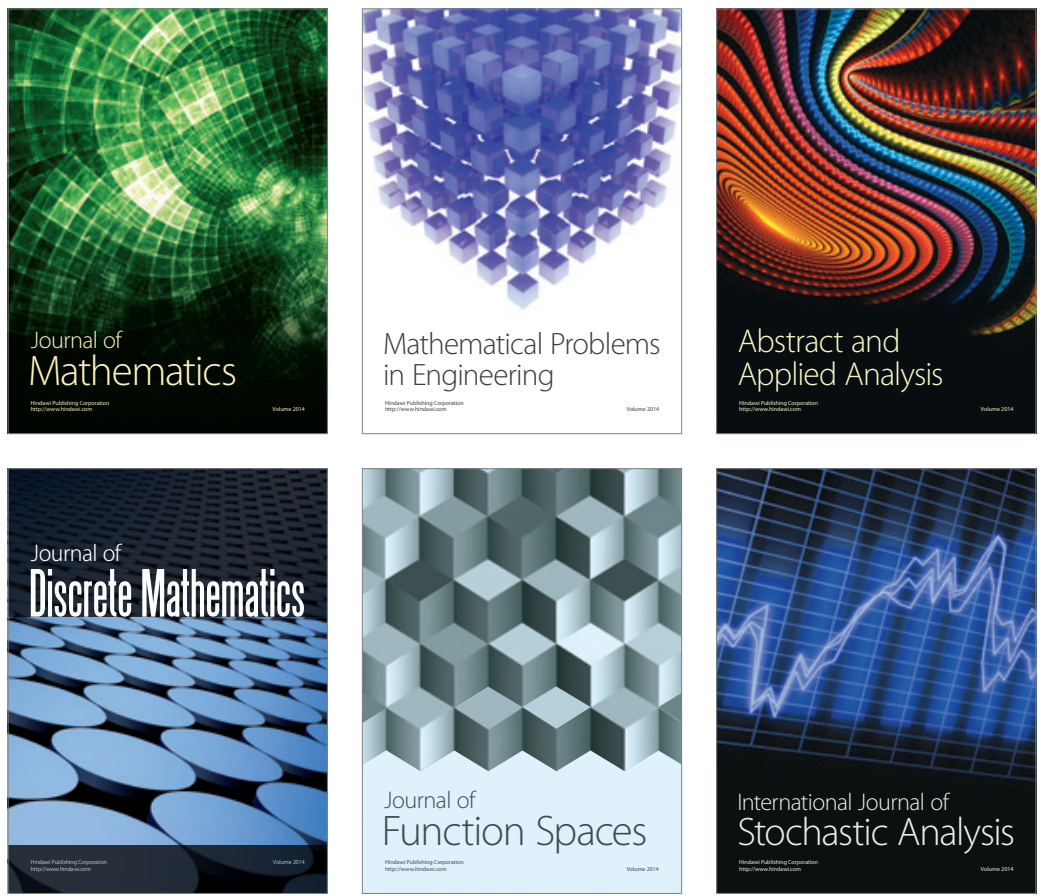

Journal of

Function Spaces

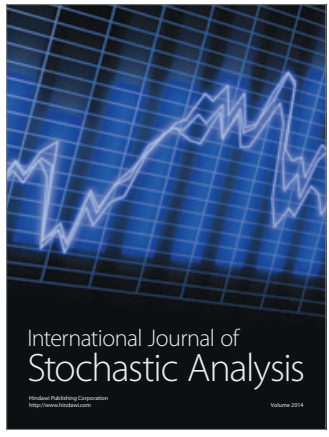

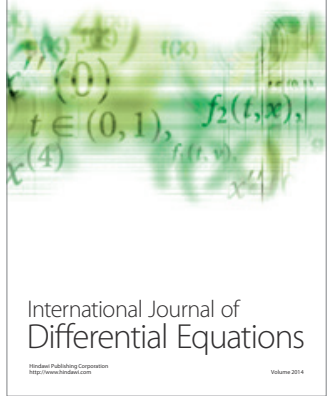
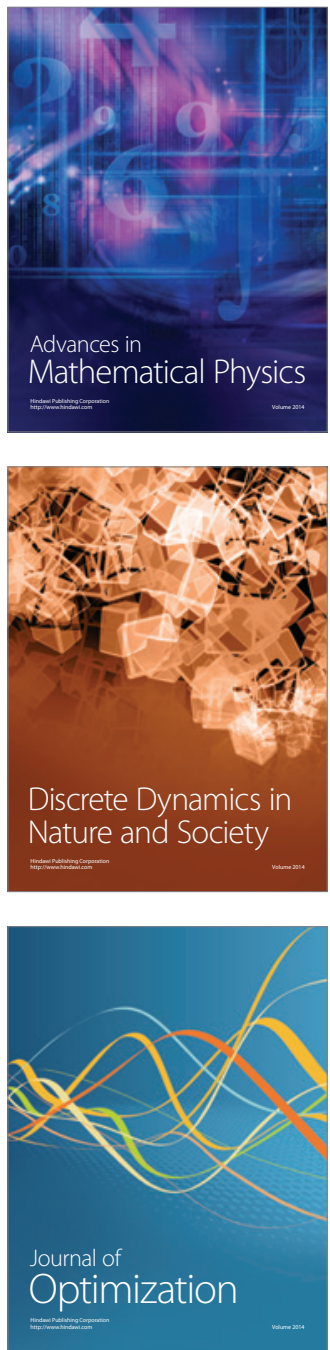